\title{
Flapping-Wing Structural Dynamics Formulation Based on a Corotational Shell Finite Element
}

\author{
Satish K. Chimakurthi* and Carlos E. S. Cesnik \\ University of Michigan, Ann Arbor, Michigan 48109 \\ and \\ Bret K. Stanford \\ U. S. Air Force Research Laboratory, Wright-Patterson Air Force Base, Dayton, Ohio 45440
}

DOI: $\underline{10.2514 / 1 . J 050494}$

\begin{abstract}
Flexible flapping wings have garnered a large amount of attention within the micro aerial vehicle community: a critical component of computational micro aerial vehicle simulations is the representation of the structural dynamics behavior of the flapping-wing structure. This paper discusses the development of a new nonlinear finite element solver that is based on a corotational approach and suitable for simulating flapping plate/shell-like wing structures undergoing small strains and large displacements/rotations. Partial verification and validation studies are presented on rectangular/elliptic wing structures to test the rigid body kinematics, nonlinear statics, and dynamics capabilities of the solver. Results obtained showed good agreement with available analytical/experimental/commercial solutions. The new structural dynamics formulation along with the numerical test cases contribute to the very limited set of tools and examples existing in the flapping-wing micro aerial vehicle literature.
\end{abstract}

$\boldsymbol{A}$
$\boldsymbol{a}$
$A_{e}$
$\boldsymbol{B}$
$\boldsymbol{B}_{\boldsymbol{b}}$
$\boldsymbol{B}_{\boldsymbol{m}}$

$\boldsymbol{C}$
$c$
$\boldsymbol{C}_{\mathrm{el}}$
$\boldsymbol{C}_{\mathrm{el}-g}$
$\boldsymbol{D}$
$D_{s}$
$\boldsymbol{d}_{\boldsymbol{e}}$
$\boldsymbol{d}_{\text {pure }}$
$\boldsymbol{d}_{\text {pure }}^{\boldsymbol{m}}$
$E$
$E_{o}$
$\boldsymbol{F}$

$\boldsymbol{F}_{\mathrm{el}}^{p}$
$\boldsymbol{F}_{\mathrm{el}-g}^{p}$
$\boldsymbol{G}$
$g$
$H$
$h$
$h_{a}$
$h_{\mathrm{root}}$
$h_{s}$
$I$
$\boldsymbol{I}_{\boldsymbol{m}}$
$\boldsymbol{J}$
$J$
$j$
$\boldsymbol{J}_{t}$
$\boldsymbol{K}_{\boldsymbol{b}}$
$\boldsymbol{K}_{\mathrm{eff}}$
$\boldsymbol{K}_{\boldsymbol{m}}$

Presented as Paper 2009-2412 at the 50th AIAA/ASME/ASCE/AHS/ASC Structures, Structural Dynamics, and Materials Conference, Palm Springs, CA, 4-7 May 2009; received 8 February 2010; revision received 27 September 2010; accepted for publication 29 September 2010. Copyright $\odot$ 2010 by Satish Kumar Chimakurthi, Carlos E. S. Cesnik, and Bret K. Stanford. Published by the American Institute of Aeronautics and Astronautics, Inc., with permission. Copies of this paper may be made for personal or internal use, on condition that the copier pay the $\$ 10.00$ per-copy fee to the Copyright Clearance Center, Inc., 222 Rosewood Drive, Danvers, MA 01923; include the code 0001-1452/11 and $\$ 10.00$ in correspondence with the CCC.

${ }^{*}$ Research Fellow, Department of Aerospace Engineering. Member AIAA.

${ }^{\dagger}$ Professor and Graduate Chair, Department of Aerospace Engineering. Associate Fellow AIAA.

${ }^{\ddagger}$ National Research Council Postdoctoral Fellow. Member AIAA.
$K_{t}$

$=$ local force vector corresponding to inertial forces due to prescribed rigid body motion

$=$ global force vector corresponding to inertial forces due to prescribed rigid body motion

$=$ matrix containing derivatives of shape functions, shear modulus

$=$ global frame

$=$ distribution function used in the thin-plate spline interpolation

$=$ discretized grid spacing

$=$ stroke amplitude due to either prescribed plunge motion or flap rotation at the wing root

$=$ chord-normalized plunge amplitude at the root (ratio of plunge amplitude at the root and chord)

$=$ plate thickness

$=$ inertial frame

$=$ identity matrix of size $3 \times 3$

$=$ Jacobian matrix associated with the transformation from Cartesian to curvilinear coordinates

$=$ torsional constant

$=$ iteration number within the Newton-Raphson iteration loop

$=$ diagonal matrix containing inverse of finite element Jacobian matrix as its components

$=$ stiffness matrix corresponding to plate bending degrees of freedom

$=$ effective stiffness matrix

$=$ stiffness matrix corresponding to membrane degrees of freedom

$=$ assembled tangent stiffness matrix in the global frame

$\boldsymbol{K}_{\mathrm{el}}^{\mathrm{dyn}} \quad=$ element local dynamic stiffness matrix

$\boldsymbol{K}_{\mathrm{el}}^{\text {shell }} \quad=$ element shell stiffness matrix

$\boldsymbol{K}_{\mathrm{el}}^{\text {shell- } p}=$ element shell stiffness matrix filtered through the projector matrix

$\boldsymbol{K}_{\mathrm{el}}^{\mathrm{ss}} \quad=$ element stress stiffening matrix

$\boldsymbol{M} \quad=$ assembled mass matrix in the global frame, typical point in the bilinear interpolation method

$\boldsymbol{M}_{\mathrm{el}} \quad=$ element local mass matrix

$\boldsymbol{M}_{\mathrm{el}-g} \quad=$ element global mass matrix

$N^{\mathrm{el}-g}=$ matrix of shape functions of size $3 \times 18$

$n=$ time level

$N_{e} \quad=$ number of finite elements 


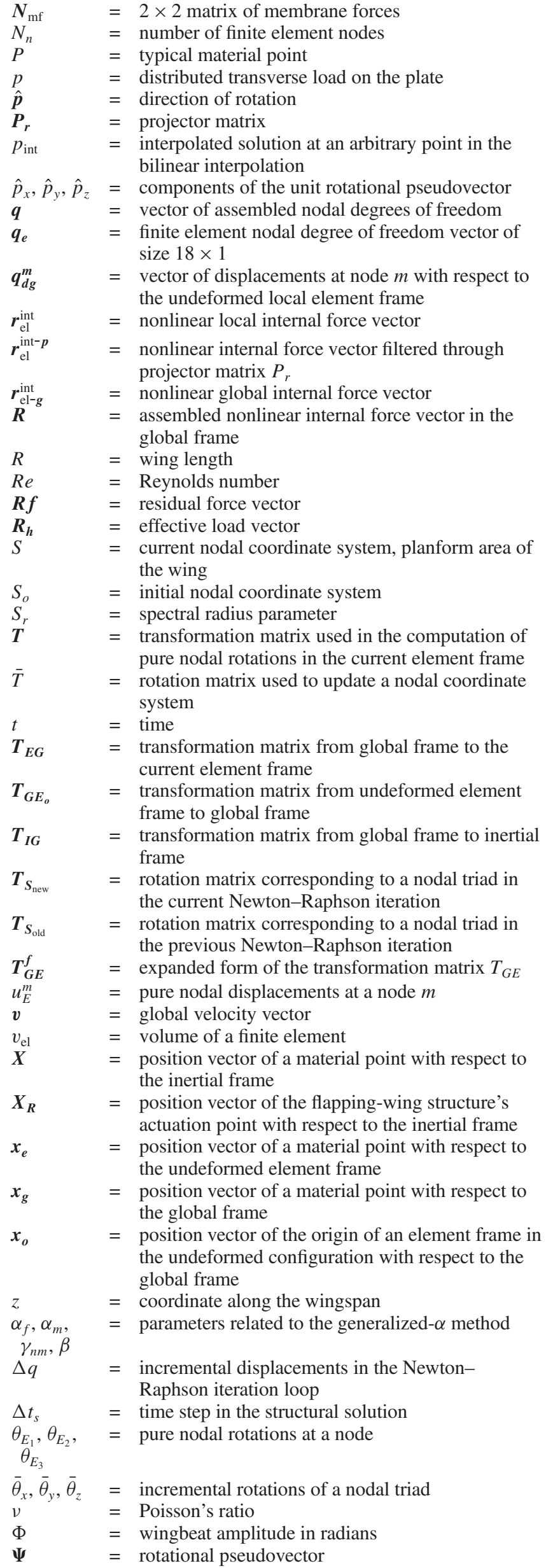

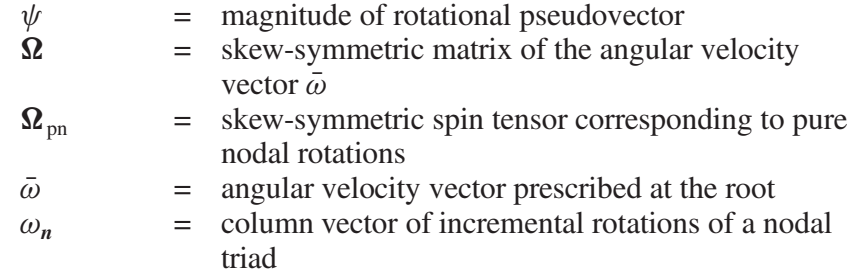

\section{Introduction}

$\mathbf{F}$ LAPPING-WING micro aerial vehicles (MAVs) are envisioned as being small (maximum dimension of $15 \mathrm{~cm}$ ), flying at low speeds $(10-15 \mathrm{~m} / \mathrm{s})$, and equipped with the capabilities of stable hover and vertical takeoff. These vehicles are basically inspired by biological flyers, including large insects and/or small birds. The mechanical properties of a typical insect wing are anisotropic because of its typical membrane-vein type configuration. It was shown that, in a majority of insect species, the spanwise bending stiffness is about one to two orders of magnitude larger than the chordwise bending stiffness [1] and, in general, the spanwise flexural stiffness scales with the third power of the wing chord while the chordwise stiffness scales with the second power of the wing chord [1]. High-speed cine and still photography and stroboscopy indicate that most biological flyers undergo orderly deformation in flight and that a controlled change of wing shape during the beat cycle may prove to be essential for the development of adequate net lift and thrust. The experimentally observed patterns of insect wing deformation indicate that they involve significant camber motions [2]. Finite element (FE) models of thin flapping wings should therefore account for such deformations, and so plate/shell elements will be appropriate to analyze them.

This work describes the development of a new shell element using the corotational (CR) approach [3-5] to analyze flapping-wing structures undergoing large (global) displacements and rotations but small strains. The key motivating factors behind the development of the CR methods are simplicity of formulation and the ability to reuse existing, very efficient, small strain, linear elements. They can also be adapted to have large strains, as shown in [6]. A key motivating factor behind the development of a new shell element is that commercial codes like MSC.Marc are not amenable to implicit coupling with an external computational fluid dynamics solver for fluid-structure interaction computations. Moreover, the proposed formulation is a fundamental step toward other developments in flapping-wing analyses. For example, if one wants to do gradient-based design, model reduction, time-periodic schemes, etc., this formulation can provide convenient Jacobians to support such analyses, while a code like MSC.Marc may not do so in an effective way.

The CR approach was previously applied by several researchers to static modeling of structures undergoing large displacements/ rotations and small deformations [4,7]. In many of the cases where the approach was applied in a dynamic context, the structures were limited to beam elements [8-13]. Applications to the dynamics of shell structures are very scarce in literature [14-17]; furthermore, their application to shell elements for flexible multibody system applications, including those of flapping wings, are even more scarce $[\underline{16}, \underline{18}, \underline{19}]$

As part of the current effort, the static CR formulation for shell elements developed previously by Khosravi et al. [4,5] is adapted to include the dynamics associated with flapping. The static element formulation of $[4,5]$ is new, in that the stiffness matrix for the shell element was developed by combining the discrete Kirchoff triangle (DKT) plate bending element [20] and the optimal triangle (OPT) membrane element [21]. The DKT element was found to be most effective among several nine-degree-of-freedom (DOF) triangular plate elements studied in [20], based on criteria-combining accuracy, computational cost, and simplicity in use. The formulation of the OPT element is based on the assumed natural deviatoric strain template [21], which is a general formulation that can produce a group of elements by assigning different values to some free parameters [4]: a special set of which gives the OPT element, which is 
insensitive to aspect ratio of the FE. Furthermore, a projector matrix [22] was applied to the shell stiffness matrix in order to correctly account for its rigid body properties. The tangent stiffness matrix was then obtained by including the effect of stress stiffening in the CR frame using the Green-Lagrange strain-displacement relations. This enables the element to account for larger rigid body structural displacements and rotations than the conventional geometrically nonlinear plate/shell elements, which use von Karman strains with respect to a fixed frame. It may be noted that, although the current work is based on many efforts that already appear in literature, the paper incorporates many disparate techniques that have not been incorporated before. Some of those include the use of the CR-totalLagrangian (CR-TL) representation of a triangular shell with multibody dynamics effects, numerical integration with the generalized- $\alpha$ method $[\underline{23}, 24]$, and (more important) the application to flappingwing structures.

The rest of the paper is organized as follows. Section II describes the entire computational structural dynamics (CSD) formulation in great detail, starting from the definitions of various coordinate systems used in the analysis, the computation of virtual work due to inertial forces, the formulation of the element tangent stiffness and mass matrices, and the time integration of the governing equations of motion using the generalized- $\alpha$ method $[23,24]$. Section III presents some verification and validation studies for the $\overline{C S D}$ solver, including examples to illustrate the rigid body kinematics, static, and dynamic response capabilities of the code. Finally, the concluding remarks are made in Sec. IV.

\section{Computational Structural Dynamics Formulation (University of Michigan Nonlinear Membrane Shell Solver)}

This section presents the theoretical formulation for the structural dynamics solution [University of Michigan Nonlinear Membrane Shell Solver (UM/NLAMS)] that was developed in this work. The geometrically nonlinear shell FE formulation developed here is based on a CR approach and has the capability to handle timedependent boundary conditions suitable for a flapping-wing problem. The nonlinear structural dynamics solution is based on a flexible multibody-type FE analysis $[3,16]$ of a flapping wing. It relies on the use of a body-fixed floating frame of reference to describe the prescribed rigid body motion and on a CR form of the TL approach [25] to account for geometric nonlinearities. The solution is implemented in the UM/NLAMS, written in Fortran 90. The CR formulation has generated a great amount of interest in the last couple of decades. A comprehensive list of references that discuss this formulation is available in [3]. The idea of this approach is to decompose the motion into rigid body and pure deformational parts through the use of a local frame at each FE, which translates and rotates with the element [26]. The components of the element's internal force vector are first calculated relative to the $\mathrm{CR}$ frame and are then transformed to a global frame using a CR transformation matrix. The $\mathrm{CR}$ frame transformation eliminates the element rigid body motion so that a linear deformation theory can be used [ 3 ] as long as the flexible deformations are small with respect to the element dimensions. Hence, its main advantage is its effectiveness for problems with small strains but large rotations [6].

The CR formulation can be applied in two different forms, as listed next:

1) In the corotational-total-Lagrangian approach (CR-TL), the reference configuration is taken as the initial configuration but translated and rotated in accordance with the motion of the corotating local system.

2) In the corotational-updated-Lagrangian approach (CR-UL), the translated and rotated configuration at the previous time step is taken as the reference configuration during the current time step [27].

The CR-TL approach is used in this work. The key motivation behind the use of it is that the stiffness matrix for the triangular shell element was originally formulated by Khosravi et al. [4] using a CR-TL approach and extensively verified/validated. The current work is an extension of their effort to include flapping-wing dynamics. Application of this method to problems concerning flapping-wing aeroelasticity are not available; however, recent studies by Relvas and Suleman $[17,28]$ reported the development of a method involving the application of CR theory to nonlinear aeroelasticity problems. Relvas and Suleman [17] discussed the coupling of a vortex-ring method with a CR structural solution of a four-noded shell FE and studied the stability of a nonlinear clamped plate subjected to low-speed airflow to illustrate the fluidstructure interaction procedure. In a subsequent effort, the same authors [28] presented the coupling of an Euler flow solver with a nonlinear CR beam FE solver and demonstrated it by studying the dynamic response of two-dimensional NACA 0012 airfoil section resting on a Winkler foundation. Two types of analyses were performed: the first assumed that the airfoil was rigid and the second assumed it to be flexible and discretized the airfoil surface

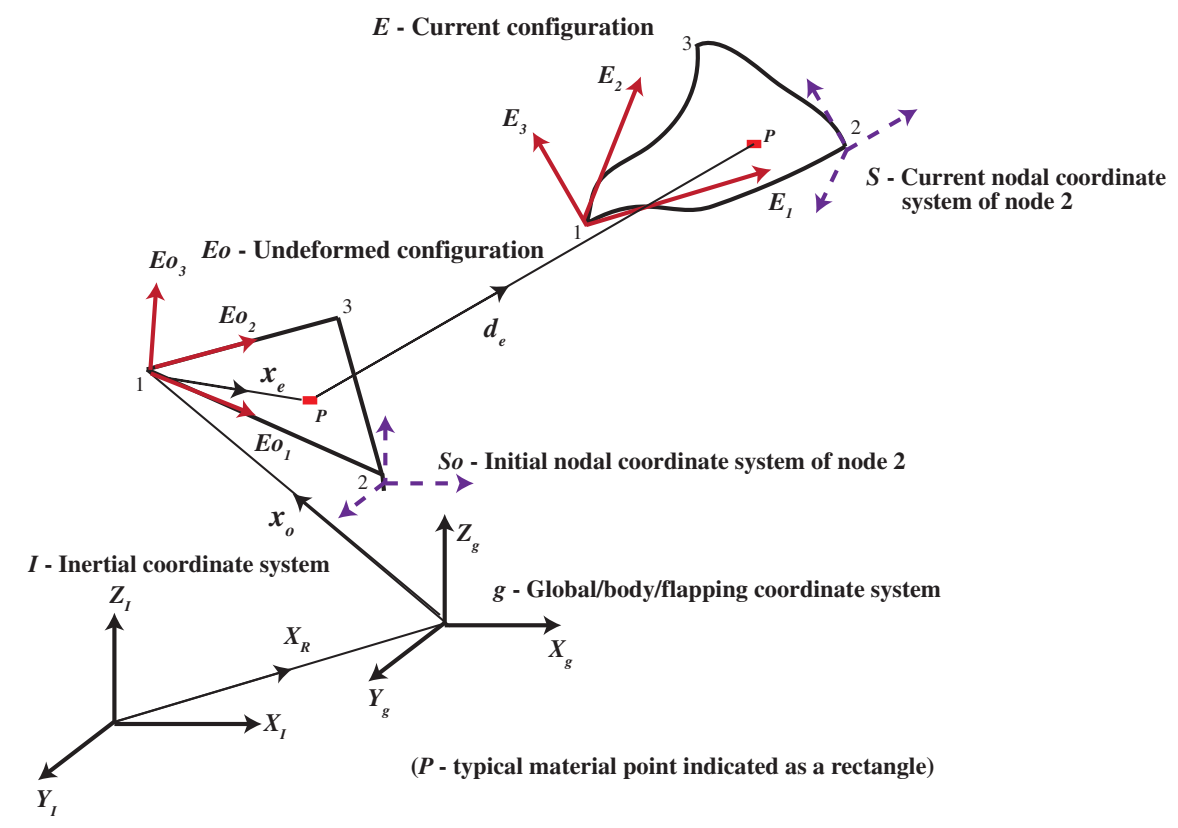

Fig. 1 A schematic showing the undeformed (initial) and deformed configurations of a typical shell element and the various coordinate systems involved in the analysis. 
with $\mathrm{CR}$ beam elements. The $\mathrm{CR}$ approach was successfully applied to analyze flutter and limit-cycle oscillations by Attar and Gordnier [29].

The three key issues identified during a literature survey in the present work concerning the use of a CR formulation are 1) the choice of a suitable local element frame, 2) the choice of a suitable element (this is especially important for triangular shells, since they are obtained as a superposition of membrane and plate models and several combinations are possible), and 3) parameterization of local and global rotations.

The first issue is discussed in $[26,30,31]$. While several alternatives have been proposed for the choice of the local element frame, for most problems where only small strains are involved, this issue is not important. However, in such a case, it is critical that the local element deformational displacements are small relative to the element dimensions. In the current work, triangular elements will be used for the FE discretization. The specific issues involved in choosing local element frames concerning the use of triangular shell elements are discussed in [7], and the choice of a suitable linear element is discussed in [32]. The development of flapping-wing dynamic FE equations of motion for thin shell structures is discussed next. The formulation is a proposed extension for flapping-wing dynamics of the static $\mathrm{CR}$ analysis of shell structures presented previously $[4,5,33]$.

The derivation of flapping-wing equations of motion using nonlinear shell FEs via a CR approach to accommodate prescribed time-dependent boundary conditions involves several key steps discussed next (note that a matrix notation is followed in the formulation).

\section{A. Definition of Coordinate Systems in Analysis}

Several coordinate systems are required to fully describe the geometry and deformation of the shell structure. Figure 1 shows a schematic of a typical triangular shell FE in its initial (undeformed) and the deformed (current) configurations. A total of $2+N_{e}+N_{n}$ (where $N_{e}$ is the number of FEs, and $N_{n}$ is the number of nodes in the discretized structure) coordinate systems are used in the analysis:

1) The first coordinate system used is an inertial frame that is always fixed in time ( $I$ in Fig. 1 ).

2) The second coordinate system used is a floating (global) frame for which the motion is known in the inertial frame by virtue of the prescribed rigid body motion of the structure ( $g$ in Fig. 1).

3 ) The third coordinate system used is $N_{e}$ CR frames (one for each element) that translate and rotate with the element as it deforms $\left(E_{o}\right.$ and $E$ in Fig. 1).

4) The fourth coordinate system used is $N_{n}$ nodal coordinate frames (one for each node) that are rigidly tied to their respective nodes and rotate with them $\left(S_{o}\right.$ and $S$ in Fig. 1$)$.

The final equations of motion are written with respect to the global frame $g$.

\section{B. Computation of Inertial Velocities and Accelerations of a Material Point}

The position (in the inertial frame) of a material point $P$ (see Fig. 1 ) in the structure is given by

$$
X=X_{R}+T_{I G} x_{g}
$$

where $\boldsymbol{X}$ is the position vector in the inertial frame, $\boldsymbol{X}_{\boldsymbol{R}}$ is the position vector of the structure's actuation point (or the origin of the flapping/ global frame) in the inertial frame, $x_{g}$ is the position vector of $P$ in the global frame, and $\boldsymbol{T}_{I G}$ is a transformation matrix from the global frame to the inertial frame. This matrix is a nonlinear function of the components of the rotational pseudovector $\boldsymbol{\Psi}$ (a unit vector that defines a finite rotation in space) [34], which defines the orientation of the global frame with respect to the inertial frame. The pseudovector is defined as

$$
\boldsymbol{\Psi}=\psi \hat{\boldsymbol{p}}
$$

where $\psi$ is the magnitude of rotation, and $\hat{\boldsymbol{p}}$ is the direction of rotation, defined as

$$
\hat{\boldsymbol{p}}=\frac{\boldsymbol{\Psi}}{\psi}=\left\{\begin{array}{l}
\hat{p}_{x} \\
\hat{p}_{y} \\
\hat{p}_{z}
\end{array}\right\}
$$

In general, both the magnitude $\psi$ and the direction of rotation $\hat{\boldsymbol{p}}$ could be time dependent. In the case where the direction of rotation is constant, the resultant motion of the tip of the pseudovector will be in a plane. If the direction of rotation changes with time, the motion of the tip will be in three dimensions. The former case is twodimensional and the latter is three-dimensional rotation $[19,34]$. The transformation matrix $\boldsymbol{T}_{\boldsymbol{I G}}$ is defined, as in [35]:

$$
\boldsymbol{T}_{\boldsymbol{I G}}=\boldsymbol{I}_{\boldsymbol{m}}+\tilde{\hat{\boldsymbol{p}}} \sin \psi+2(\tilde{\hat{\boldsymbol{p}}})^{2} \sin ^{2} \frac{\psi}{2}
$$

where $\boldsymbol{I}_{m}$ is a $3 \times 3$ identity matrix, and the tilde indicates a skewsymmetric matrix. The position vector of the material point with respect to the global coordinate system $\boldsymbol{x}_{g}$ given in Eq. (1) can be written as

$$
x_{g}=x_{o}+T_{G E_{o}}\left(x_{e}+d_{e}\right)
$$

where $x_{\boldsymbol{o}}$ is the position vector of the origin of an element frame in the undeformed configuration with respect to the global frame expressed as components in the global frame, $\boldsymbol{T}_{\boldsymbol{G} \boldsymbol{E}_{o}}$ is the transformation matrix from undeformed element frame to the global frame, $\boldsymbol{x}_{e}$ is the position vector of the point with respect to the undeformed element frame, and $\boldsymbol{d}_{\boldsymbol{e}}$ is the vector of displacements of the point with respect to the same. The position vector of the material point in the inertial frame given in Eq. (1) then becomes

$$
X=X_{R}+T_{I G} x_{o}+T_{I G} T_{G E_{o}} x_{e}+T_{I G} T_{G E_{o}} d_{e}
$$

The time derivative of the transformation matrix in Eq. (4) is [36]:

$$
\dot{T}_{I G}=\Omega T_{I G}
$$

where $\boldsymbol{\Omega}$ is a skew-symmetric matrix of the angular velocity vector $\overline{\boldsymbol{\omega}}$ prescribed at the wing root. The velocity and acceleration of the material point can then be computed by successive differentiation of the position vector in Eq. (6) and are given as

$$
\begin{aligned}
\dot{X} & =\dot{X}_{R}+\Omega T_{I G} x_{o}+\Omega T_{I G} T_{G E_{o}} x_{e}+\Omega T_{I G} T_{G E_{o}} d_{e} \\
& +T_{I G} T_{G E_{o}} \dot{d}_{e} \\
\ddot{X} & =\ddot{X}_{R}+(\dot{\Omega}+\Omega \Omega) T_{I G}\left(x_{o}+T_{G E_{o}} x_{e}+T_{G E_{o}} d_{e}\right) \\
& +2 \Omega T_{I G} T_{G E_{o}} \dot{d}_{e}+T_{I G} T_{G E_{o}} \ddot{d}_{e}
\end{aligned}
$$

\section{Computation of Virtual Work due to Inertial Forces}

The virtual work due to inertial forces for an element is given by

$$
\delta W=\rho_{s} \int_{v_{\mathrm{el}}} \delta \boldsymbol{X}^{\boldsymbol{T}} \ddot{\boldsymbol{X}} \mathrm{d} v_{\mathrm{el}}
$$

where $\delta \boldsymbol{X}$ is the variation of the position vector; that is,

$$
\delta \boldsymbol{X}=\boldsymbol{T}_{I G} \boldsymbol{T}_{G E_{o}} \delta \boldsymbol{d}_{e}
$$

and $v_{\mathrm{el}}$ is the volume of the element. The vector of displacements $\boldsymbol{d}_{\boldsymbol{e}}$ can be approximated as

$$
d_{e}=N q_{e}
$$

where $N$ is a matrix of shape functions of size $3 \times 18$, and $\boldsymbol{q}_{e}$ is the FE nodal DOF vector of size $18 \times 1$ with respect to the undeformed element frame. Equation (11) is derived from Eq. (6) by following the regular variational approach. In the variational process, the first, second, and third terms in Eq. (6) vanish, since they are either prescribed or constant. For example, $\boldsymbol{X}_{\boldsymbol{R}}$ is the position vector of the structure's actuation point with respect to the inertial frame. This is a 
prescribed quantity in this work; hence, the variation of this term is simply zero. The variation of the position vector now becomes

$$
\delta X=T_{I G} T_{G E_{o}} N \delta q_{e}
$$

The acceleration vector can be written as

$$
\begin{aligned}
\ddot{X} & =\ddot{X}_{R}+(\dot{\Omega}+\Omega \Omega) T_{I G}\left(x_{o}+T_{G E_{o}} x_{e}+T_{G E_{o}} N q_{e}\right) \\
& +2 \Omega T_{I G} T_{G E_{o}} N \dot{q}_{e}+T_{I G} T_{G E_{o}} N \ddot{q}_{e}
\end{aligned}
$$

Using the previous terms, the virtual work expression in Eq. (10) now becomes

$$
\begin{aligned}
\delta W & =\rho_{s} \int_{v_{\mathrm{el}}}\left\{\delta \boldsymbol{q}_{e}^{T} N^{T} \boldsymbol{T}_{G E_{o}}^{T} \boldsymbol{T}_{I G}^{T} \ddot{X}_{R}+\delta \boldsymbol{q}_{e}^{T} N^{T} \boldsymbol{T}_{G E_{o}}^{T} \boldsymbol{T}_{I G}^{T}(\dot{\boldsymbol{\Omega}}\right. \\
& +\boldsymbol{\Omega} \boldsymbol{\Omega}) \boldsymbol{T}_{I G} \boldsymbol{x}_{o}+\delta \boldsymbol{q}_{e}^{T} \boldsymbol{N}^{T} \boldsymbol{T}_{G E_{o}}^{T} \boldsymbol{T}_{I G}^{T}(\dot{\boldsymbol{\Omega}}+\Omega \Omega) \boldsymbol{T}_{I G} \boldsymbol{T}_{G E_{o}} \boldsymbol{x}_{e} \\
& +\delta \boldsymbol{q}_{e}^{T} \boldsymbol{N}^{T} \boldsymbol{T}_{G E_{o}}^{T} \boldsymbol{T}_{I G}^{T}(\dot{\boldsymbol{\Omega}}+\boldsymbol{\Omega}) \boldsymbol{T}_{I G} \boldsymbol{T}_{G E_{o}} N \boldsymbol{q}_{e} \\
& +2 \delta \boldsymbol{q}_{e}^{T} \boldsymbol{N}^{T} \boldsymbol{T}_{G E_{o}}^{T} \boldsymbol{T}_{I G}^{T} \boldsymbol{\Omega} \boldsymbol{T}_{I G} \boldsymbol{T}_{G E_{o}} \boldsymbol{N} \dot{\boldsymbol{q}}_{e} \\
& \left.+\delta \boldsymbol{q}_{e}^{T} \boldsymbol{N}^{T} \boldsymbol{T}_{G E_{o}}^{T} \boldsymbol{T}_{I G}^{T} \boldsymbol{T}_{I G} \boldsymbol{T}_{G E_{o}} \boldsymbol{N} \ddot{q}_{e}\right\} \mathrm{d} v_{\mathrm{el}}
\end{aligned}
$$

From this expression, the element local mass matrix, gyroscopic damping matrix, dynamic stiffness matrix, and the inertial contribution to the force vector are given by

$$
\begin{aligned}
& \boldsymbol{M}_{\mathrm{el}}=\rho_{s} \int_{v_{\mathrm{el}}}\left\{\boldsymbol{N}^{\boldsymbol{T}} \boldsymbol{T}_{\boldsymbol{G} \boldsymbol{E}_{o}}^{\boldsymbol{T}} \boldsymbol{T}_{\boldsymbol{I G}}^{\boldsymbol{T}} \boldsymbol{T}_{\boldsymbol{I} \boldsymbol{G}} \boldsymbol{T}_{\boldsymbol{G} \boldsymbol{E}_{\boldsymbol{o}}} \boldsymbol{N}\right\} \mathrm{d} v_{\mathrm{el}} \\
& \boldsymbol{C}_{\mathrm{el}}=2 \rho_{s} \int_{v_{\mathrm{el}}}\left\{\boldsymbol{N}^{T} \boldsymbol{T}_{\boldsymbol{G} \boldsymbol{E}_{o}}^{T} \boldsymbol{T}_{\boldsymbol{I}}^{T} \boldsymbol{\Omega} \boldsymbol{T}_{\boldsymbol{I G}} \boldsymbol{T}_{\boldsymbol{G} \boldsymbol{E}_{o}} \boldsymbol{N}\right\} \mathrm{d} v_{\mathrm{el}} \\
& \boldsymbol{K}_{\mathrm{el}}^{\mathrm{dyn}}=\rho_{s} \int_{v_{\mathrm{el}}}\left\{\boldsymbol{N}^{\boldsymbol{T}} \boldsymbol{T}_{\boldsymbol{G} \boldsymbol{E}_{o}}^{\boldsymbol{T}} \boldsymbol{T}_{\boldsymbol{I} \boldsymbol{G}}^{T}(\dot{\boldsymbol{\Omega}}+\boldsymbol{\Omega} \boldsymbol{\Omega}) \boldsymbol{T}_{\boldsymbol{I} \boldsymbol{G}} \boldsymbol{T}_{\boldsymbol{G} \boldsymbol{E}_{o}} \boldsymbol{N}\right\} \mathrm{d} v_{\mathrm{el}} \\
& F_{\mathrm{el}}^{p}=-\rho_{s} \int_{v_{\mathrm{el}}}\left\{N^{T} T_{G E_{o}}^{T} T_{I G}^{T} \ddot{X}_{R}+N^{T} T_{G E_{o}}^{T} T_{I G}^{T}(\dot{\Omega}+\Omega \Omega) T_{I G} x_{o}\right. \\
& \left.+\boldsymbol{N}^{\boldsymbol{T}} \boldsymbol{T}_{\boldsymbol{G} E_{o}}^{T} \boldsymbol{T}_{I G}^{T}(\dot{\boldsymbol{\Omega}}+\boldsymbol{\Omega} \boldsymbol{\Omega}) \boldsymbol{T}_{\boldsymbol{I G}} \boldsymbol{T}_{\boldsymbol{G} E_{o}} \boldsymbol{x}_{e}\right\} \mathrm{d} v_{\mathrm{el}}
\end{aligned}
$$

These equations are numerically integrated using a seven-point Gauss quadrature [37]. The element mass matrix in Eq. (16) is consistent. The damping matrix in Eq. (17) is a skew-symmetric matrix arising from Coriolis forces. The stiffness matrix in Eq. (18) is a dynamic term representing the coupling effect between the large rigid body motions and the elastic motions. The elastic portion of the stiffness matrix will be discussed subsequently. The force vector in Eq. (19) is due to the prescribed rigid body motion (inertial contribution that initially appears with a +ve sign on the left-hand side of the equations of motion but is brought to the right with a -ve sign). The first term arises from rigid body translational motion. The second and the third terms arise from rigid body angular and centrifugal accelerations. A fourth term in the forcing vector will arise due to aerodynamic loading.

\section{Element Local Deformations (Corotational Approach)}

As mentioned previously, the static CR formulation of a shell element, as described in $[\underline{4}, \underline{5}, \underline{33}]$, is used in this work. While full details of the approach are provided in those references, a brief overview of it is presented here while extensively quoting from [4]. Referring to Fig. 1, the origin of the undeformed element frame $E_{o}$ is chosen at node 1 of the element, and the axis $E_{o_{1}}$ (i.e., the local $x$ axis) is chosen as the line joining nodes 1 and 2 . The axis $E_{o_{3}}$ (the local $z$ axis) is the normal to the element midplane containing the nodes 1,2 , and 3. The axis $E_{\mathrm{O}_{2}}$ (the local $y$ axis) then defines a Cartesian righthanded coordinate system. The coordinate system denoted by $E$ is the element CR system defined in a similar fashion but in the current or deformed configuration. The nodal coordinate systems are denoted by $S_{o}$ and $S$ in the undeformed and deformed configurations, respectively (shown only for node 2 in Fig. 1 for clarity). The orientation of $S_{o}$ is arbitrary and is chosen to be parallel to the inertial frame in this work. The coordinate system $S$ in the current configuration is obtained by updating its transformation matrix, which defines the current orientation of the node in the global system. This is done after every Newton-Raphson iteration using the following expression:

$$
\boldsymbol{T}_{S_{\text {new }}}=\overline{\boldsymbol{T}} \boldsymbol{T}_{S_{\text {old }}}
$$

where

$$
\begin{gathered}
\overline{\boldsymbol{T}}=\boldsymbol{I}_{\boldsymbol{m}}+\frac{\tilde{\boldsymbol{\omega}}_{\boldsymbol{n}}+0.5 \tilde{\boldsymbol{\omega}}_{n}^{2}}{1+0.25\left|\boldsymbol{\omega}_{\boldsymbol{n}}\right|^{2}} \\
\boldsymbol{\omega}_{n}=\left[\begin{array}{lll}
\bar{\theta}_{x} & \bar{\theta}_{y} & \bar{\theta}_{z}
\end{array}\right]^{T} \\
\tilde{\boldsymbol{\omega}}_{n}=\left[\begin{array}{ccc}
0 & -\bar{\theta}_{z} & \bar{\theta}_{y} \\
\bar{\theta}_{z} & 0 & -\bar{\theta}_{x} \\
-\bar{\theta}_{y} & \bar{\theta}_{x} & 0
\end{array}\right]
\end{gathered}
$$

The $\bar{\theta}$ quantities are the incremental rotations of triad $S$ computed in the global coordinate system during the previous iteration. Once the nodal coordinate systems in the current configuration are obtained, the next step is the computation of the pure deformations (both displacements and rotations) in the local coordinate system $E$. Pure nodal displacements at a node $m$ in $E$ may be expressed by the relation,

$$
\boldsymbol{u}_{\boldsymbol{E}}^{\boldsymbol{m}}=\left\{\begin{array}{l}
u_{E_{1}}^{m} \\
u_{E_{2}}^{m} \\
u_{E_{3}}^{m}
\end{array}\right\}=\boldsymbol{T}_{\boldsymbol{E} \boldsymbol{G}}\left(\boldsymbol{q}_{\boldsymbol{d} g}^{\boldsymbol{m}}+\boldsymbol{x}_{\boldsymbol{o}}^{\boldsymbol{m}}-\boldsymbol{q}_{\boldsymbol{d} g}^{1}-\boldsymbol{x}_{\boldsymbol{o}}^{1}\right)-\boldsymbol{x}_{\boldsymbol{e}}^{\boldsymbol{m}}
$$

where $m=1,2$, and 3. $\boldsymbol{T}_{\boldsymbol{E} \boldsymbol{G}}$ is a transformation matrix from the global frame to the current element frame, $\boldsymbol{q}_{d g}^{m}$ is the displacement vector of a node $m$ in the global frame, and $\boldsymbol{x}_{\boldsymbol{o}}^{\boldsymbol{m}}$ is the position vector of node $m$ in the undeformed element configuration expressed in the global frame. The variable $\boldsymbol{x}_{\boldsymbol{o}}^{1}$ is equal to $\boldsymbol{x}_{\boldsymbol{o}}$, introduced in Eq. (5). Pure nodal rotations expressed in $E$ are equal to the components of a skew-symmetric matrix spin tensor defined as

$$
\boldsymbol{\Omega}_{\boldsymbol{p} \boldsymbol{n}}=\left[\begin{array}{ccc}
0 & -\theta_{E_{3}} & \theta_{E_{2}} \\
\theta_{E_{3}} & 0 & -\theta_{E_{1}} \\
-\theta_{E_{2}} & \theta_{E_{1}} & 0
\end{array}\right]
$$

This tensor is found by using the following expressions:

$$
\begin{gathered}
\boldsymbol{\Omega}_{p n}=2\left(\boldsymbol{T}-\boldsymbol{I}_{\boldsymbol{m}}\right)\left(\boldsymbol{T}+\boldsymbol{I}_{\boldsymbol{m}}\right)^{-1} \\
\boldsymbol{T}=\boldsymbol{T}_{\boldsymbol{E} G} \boldsymbol{T}_{S_{\text {new }}} \boldsymbol{T}_{\boldsymbol{G} E_{o}}
\end{gathered}
$$

where the matrices $\boldsymbol{T}_{\boldsymbol{E} G}$ and $\boldsymbol{T}_{\boldsymbol{G} \boldsymbol{E}_{o}}^{T}$ transform the components of a vector in the global frame into those in deformed $\mathrm{CR}$ and undeformed $\mathrm{CR}$ frames, respectively. The vector of pure deformations at a node is given by

$$
\boldsymbol{d}_{\text {pure }}^{\boldsymbol{m}}=\left[\begin{array}{llllll}
u_{E_{1}}^{m} & u_{E_{2}}^{m} & u_{E_{3}}^{m} & \theta_{E_{1}}^{m} & \theta_{E_{2}}^{m} & \theta_{E_{3}}^{m}
\end{array}\right]^{T}
$$

The vector of pure element deformations is obtained by combining such vectors at all three nodes of the element and is given by

$$
\boldsymbol{d}_{\text {pure }}=\left(\begin{array}{l}
d_{\text {pure }}^{1} \\
d_{\text {pure }}^{2} \\
d_{\text {pure }}^{3}
\end{array}\right)
$$




\section{E. Element Stiffness Matrix/Nonlinear Internal Force Vector}

A three-node triangular shell element involving an optimal membrane element (OPT) [21] and a DKT plate bending element [20] presented in $[4,5]$ is used in this work. Both of these elements are described briefly below:

1) The DKT element formulation is based on discrete Kirchoff theory of thin plates. It begins with independent fields for lateral displacement and for rotation of a midsurface normal line, like in Mindlin elements [38]. In this approach, the strain energy contribution due to shear stresses is neglected, and the normality to the midsurface is enforced at discrete points at the element edges. With these constraints imposed, a 9-DOF element is obtained with two rotations and a displacement at each of the element nodes. Full details of the DKT formulation are given in [38]. As a consequence of the process by which the element is derived, the transverse displacement is not explicitly defined in the interior of the element. Hence, the shape functions required to form the mass, damping, dynamic stiffness, and the stress stiffening matrix (discussed later) along with the force vector are not available. This problem may be overcome by borrowing shape functions from other similar elements. Following [4], for the displacement interpolation, the shape functions corresponding to a Bazeley, Cheung, Irons, and Zienkiewicz plate element [37] are used. The stiffness matrix (of size $9 \times 9$ ) corresponding to the plate bending DOF can be written as [4]

$$
\boldsymbol{K}_{b}=\int \boldsymbol{B}_{b}^{T} \boldsymbol{D} \boldsymbol{B}_{b} \mathrm{~d} A_{e}
$$

where $\boldsymbol{B}_{b}$ is the strain-nodal displacement matrix corresponding to bending deformation, $\boldsymbol{D}$ is the bending stiffness matrix, and $A_{e}$ is the area of the triangular FE.

2) The OPT element is termed optimal because, for any arbitrary aspect ratio, its response for in-plane pure bending is exact. Like the DKT element, the OPT element is based on an assumption on strains, and so the shape functions are borrowed from another triangular membrane element (linear strain triangle-retrofitted) with the same DOFs as that of the OPT element. The stiffness matrix (of size $9 \times 9$ ) corresponding to the membrane DOF is [4]

$$
\boldsymbol{K}_{m}=\int \boldsymbol{B}_{m}^{T} \boldsymbol{A} \boldsymbol{B}_{m} \mathrm{~d} A_{e}
$$

where $\boldsymbol{B}_{\boldsymbol{m}}$ is the strain-nodal displacement matrix corresponding to membrane deformation, and $\boldsymbol{A}$ is the stretching stiffness matrix.

The DKT and the OPT element stiffness matrices are combined to form the final shell stiffness matrix of the element and are further modified to include the membrane-bending coupling effect for laminated composite plates:

$$
\boldsymbol{K}_{\mathrm{el}}^{\text {shell }}=\left[\begin{array}{cc}
\boldsymbol{K}_{\boldsymbol{m}} & \int \boldsymbol{B}_{\boldsymbol{m}}^{T} \boldsymbol{B} \boldsymbol{B}_{\boldsymbol{b}} \mathrm{d} A_{e} \\
\int \boldsymbol{B}_{\boldsymbol{b}}^{\boldsymbol{T}} \boldsymbol{B} \boldsymbol{B}_{m} \mathrm{~d} A_{e} & \boldsymbol{K}_{\boldsymbol{b}}
\end{array}\right]
$$

where $\boldsymbol{B}$ is the membrane-bending coupling stiffness matrix. More details of the stiffness matrices, including the definition of the individual terms, are presented in [4].

The effect of nonlinear stress stiffening is added to the CR formulation by including a geometric stiffness matrix [38]. The expression for stress stiffening is obtained by considering the work done by the membrane forces (computed from the solution of the previous iteration) as they act through displacements associated with small lateral and in-plane deflections. The final expression for the stress stiffening matrix is given by

$$
\boldsymbol{K}_{\mathrm{el}}^{\mathrm{ss}}=\int \boldsymbol{G}^{\boldsymbol{T}}\left[\begin{array}{lll}
\boldsymbol{N}_{\mathrm{mf}} & 0_{2 \times 2} & 0_{2 \times 2} \\
0_{2 \times 2} & \boldsymbol{N}_{\mathrm{mf}} & 0_{2 \times 2} \\
0_{2 \times 2} & 0_{2 \times 2} & \boldsymbol{N}_{\mathrm{mf}}
\end{array}\right] \boldsymbol{G} \mathrm{d} A
$$

The submatrix $N_{\text {mf }}$ of size $2 \times 2$, for which the components are the membrane forces, is the same as $\tilde{\boldsymbol{N}}$, defined in [4]. More details on the derivation of this expression along with a definition of the individual terms are given in $[\underline{4}, \underline{38}]$.
Using the pure element deformations in Eq. (29), the nonlinear internal force vector is computed using the local element shell and dynamic stiffness matrices [after rearranging them according to desired order of DOFs, as in Eq. (29)], as

$$
\boldsymbol{r}_{\mathrm{el}}^{\mathrm{int}}=\left(\boldsymbol{K}_{\mathrm{el}}^{\text {shell }}+\boldsymbol{K}_{\mathrm{el}}^{\mathrm{dyn}}\right) \boldsymbol{d}_{\text {pure }}
$$

Since the pure deformations obtained in Eq. (29) may not really be pure (i.e., without any rigid body components), a projector matrix $\boldsymbol{P}_{\boldsymbol{r}}$ can be introduced to bring the nonequilibrated internal force vector into equilibrium. The details of the projection are beyond the scope of this paper. The readers are referred to [22] for more details. The local element stiffness matrix computed in Eq. (32) and the internal force vector computed in Eq. (34) are filtered through the projector matrix as follows:

$$
\begin{gathered}
\boldsymbol{K}_{\mathrm{el}}^{\text {shell }-\boldsymbol{p}}=\boldsymbol{P}_{\boldsymbol{r}}^{T} \boldsymbol{K}_{\mathrm{el}}^{\text {shell }} \boldsymbol{P}_{\boldsymbol{r}} \\
\boldsymbol{r}_{\mathrm{el}}^{\mathrm{int}-\boldsymbol{p}}=\boldsymbol{P}_{r}^{T} \boldsymbol{K}_{\mathrm{el}}^{\text {shell }} \boldsymbol{d}_{\text {pure }}
\end{gathered}
$$

In computing the projection of the internal force vector, as shown in Eq. (36), the contribution due to the dynamic stiffness matrix is excluded. At this point, if the membrane forces are expected to be significant, the stress stiffness matrix obtained in Eq. (33) should be added to the projected local element stiffness matrix in Eq. (35).

Having obtained the stiffness and mass matrices along with the internal force vector in the element frame, they are all transformed into the global frame before the FE assembly process. The transformation of the element local stiffness matrix, which includes both the elastic and the dynamic stiffness terms, is given as

$$
\boldsymbol{K}_{\mathrm{el}-g}^{\text {shelldyn- } p}=\left(\boldsymbol{T}_{G E}^{f}\right)\left(\boldsymbol{K}_{\mathrm{el}}^{\mathrm{shell}-p}+\boldsymbol{K}_{\mathrm{el}}^{\mathrm{dyn}}\right)\left(\boldsymbol{T}_{G E}^{f}\right)^{T}
$$

where the transformation matrix $T_{G E}^{f}$ (previously mentioned) is an expanded form of $T_{G E}$ (which is a transpose of $T_{E G}$, defined earlier) used to accommodate the transformation of all 18 DOFs of the element. The subscript $-g$ indicates that the corresponding element matrix operates on the global DOFs. The superscript $f$ indicates full/ expanded. Similarly, the element mass and the gyroscopic matrices given in Eqs. (16) and (17), respectively, are transformed into the global frame as follows:

$$
\begin{gathered}
M_{\mathrm{el}-g}=T_{G E_{o}} M_{\mathrm{el}} \boldsymbol{T}_{G E_{o}}^{T} \\
C_{\mathrm{el}-g}=T_{G E_{o}} C_{\mathrm{el}} \boldsymbol{T}_{G E_{o}}^{T}
\end{gathered}
$$

Furthermore, the element internal force and the prescribed-motion force vectors given in Eqs. (19) and (36), respectively, are transformed to the global frame as

$$
\begin{gathered}
\boldsymbol{r}_{\mathrm{el}-g}^{\mathrm{int}}=\boldsymbol{T}_{\boldsymbol{G}}^{f} \boldsymbol{r}_{\mathrm{el}}^{\mathrm{int}-p} \\
\boldsymbol{F}_{\mathrm{el}-g}^{p}=\boldsymbol{T}_{\boldsymbol{G} \boldsymbol{E}_{o}}^{f} \boldsymbol{F}_{\mathrm{el}}^{p}
\end{gathered}
$$

The global element mass, stiffness, and gyroscopic damping matrices given in Eqs. (37-39), respectively, and the element global internal force and prescribe $\mathrm{d}$-motion force vectors given in Eqs. (40) and (41), respectively, are assembled for the entire structure to form global matrices/vectors. The global mass, tangent stiffness, and damping matrices are denoted as $\boldsymbol{M}, \boldsymbol{K}_{\boldsymbol{t}}$ (assembled form of $\boldsymbol{K}_{\mathrm{el}-g}^{\text {sheldyn- } \boldsymbol{p}}$ matrix for the entire structure), and $\boldsymbol{C}$, respectively, while the global internal and the total force vectors are denoted as $\boldsymbol{R}$ and $\boldsymbol{F}$, respectively.

\section{F. Direct Time Integration of University of Michigan Nonlinear Membrane Shell Solver Governing Equations}

The nonlinear structural dynamics FE governing equations of motion can be written as 


$$
M a+C v+R(q)=F
$$

where $\boldsymbol{q}$ is the nodal DOF vector in the global frame, and $\boldsymbol{v}$ and $\boldsymbol{a}$ are the global velocity and acceleration vectors, respectively. In this work, the numerical integration of the governing equations was performed using either the Newmark or the generalized- $\alpha$ methods $[\underline{23}, 24]$. Reference [23] discussed the application of the generalized$\alpha$ scheme for linear problems. In this work, it is extended to solve the nonlinear equations of motion in a predictor-corrector-type of framework similar to the one described for the Newmark method in [39]. The generalized- $\alpha$ method discussed in [23] solves secondorder differential equations for a discrete time step $n$, using the standard Newmark relations to update the displacements and velocities, as shown next:

$$
\begin{gathered}
\boldsymbol{q}_{\boldsymbol{n}+1}=\boldsymbol{q}_{\boldsymbol{n}}+\Delta t_{s} \boldsymbol{v}_{\boldsymbol{n}}+\Delta t_{s}^{2}\left[\left(\frac{1}{2}-\beta\right) \boldsymbol{a}_{\boldsymbol{n}}+\beta \boldsymbol{a}_{\boldsymbol{n}+1}\right] \\
\boldsymbol{v}_{\boldsymbol{n}+1}=\boldsymbol{v}_{\boldsymbol{n}}+\Delta t_{s}\left[\left(1-\gamma_{n m}\right) \boldsymbol{a}_{\boldsymbol{n}}+\gamma_{n m} \boldsymbol{a}_{\boldsymbol{n}+1}\right]
\end{gathered}
$$

The balance equation is given by

$$
M a_{n+1-\alpha_{m}}+C v_{n+1-\alpha_{f}}+R_{n+1-\alpha_{f}}=\boldsymbol{F}\left(t_{n+1-\alpha_{f}}\right)
$$

where

$$
\begin{aligned}
& \boldsymbol{a}_{\boldsymbol{n}+1-\alpha_{m}}=\left(1-\alpha_{m}\right) \boldsymbol{a}_{\boldsymbol{n}+1}+\alpha_{m} \boldsymbol{a}_{\boldsymbol{n}} \\
& \boldsymbol{v}_{\boldsymbol{n}+1-\alpha_{f}}=\left(1-\alpha_{f}\right) \boldsymbol{v}_{\boldsymbol{n}+1}+\alpha_{f} \boldsymbol{v}_{n} \\
& \boldsymbol{R}_{\boldsymbol{n}+1-\alpha_{f}}=\left(1-\alpha_{f}\right) \boldsymbol{R}_{\boldsymbol{n}+1}+\alpha_{f} \boldsymbol{R}_{\boldsymbol{n}} \\
& \boldsymbol{F}\left(\boldsymbol{t}_{\boldsymbol{n}+1-\alpha_{f}}\right)=\left(1-\alpha_{f}\right) \boldsymbol{F}_{\boldsymbol{n}+1}+\alpha_{f} \boldsymbol{F}_{\boldsymbol{n}}
\end{aligned}
$$

Substituting the relations in Eq. (46) into the balance equation in Eq. (45) gives

$$
\begin{array}{r}
\boldsymbol{M}\left[\left(1-\alpha_{m}\right) \boldsymbol{a}_{\boldsymbol{n}+1}+\alpha_{m} \boldsymbol{a}_{\boldsymbol{n}}\right]+\boldsymbol{C}\left[\left(1-\alpha_{f}\right) \boldsymbol{v}_{\boldsymbol{n}+1}+\alpha_{f} \boldsymbol{v}_{\boldsymbol{n}}\right] \\
\quad+\left(1-\alpha_{f}\right) \boldsymbol{R}_{\boldsymbol{n}+1}+\alpha_{f} \boldsymbol{R}_{\boldsymbol{n}}=\left(1-\alpha_{f}\right) \boldsymbol{F}_{\boldsymbol{n}+1}+\alpha_{f} \boldsymbol{F}_{\boldsymbol{n}}
\end{array}
$$

Using the Newmark update relation of displacements in Eq. (43), the accelerations become

$$
\begin{gathered}
\boldsymbol{a}_{n+1}=\frac{1}{\beta \Delta t_{s}^{2}}\left[\boldsymbol{q}_{\boldsymbol{n}+1}-\boldsymbol{q}_{\boldsymbol{n}}-\Delta t_{s} \boldsymbol{v}_{\boldsymbol{n}}-\Delta t_{s}^{2}\left(\frac{1}{2}-\beta\right) \boldsymbol{a}_{\boldsymbol{n}}\right] \\
\boldsymbol{a}_{\boldsymbol{n}+1}=\frac{1}{\beta \Delta t_{s}^{2}}\left[\delta \boldsymbol{q}-\Delta t_{s} \boldsymbol{v}_{\boldsymbol{n}}-\Delta t_{s}^{2}\left(\frac{1}{2}-\beta\right) \boldsymbol{a}_{\boldsymbol{n}}\right]
\end{gathered}
$$

Substituting this into the velocity update relation equation (44) gives

$$
\begin{gathered}
\boldsymbol{v}_{\boldsymbol{n}+1}=\boldsymbol{v}_{\boldsymbol{n}}+\Delta t_{s}\left(1-\gamma_{n m}\right) \boldsymbol{a}_{\boldsymbol{n}}+\frac{\gamma_{n m}}{\beta \Delta t_{s}} \boldsymbol{\delta} \boldsymbol{q}-\frac{\gamma_{n m}}{\beta} \boldsymbol{v}_{\boldsymbol{n}} \\
-\frac{\gamma_{n m} \Delta t_{s}}{\beta}\left(\frac{1}{2}-\beta\right) \boldsymbol{a}_{\boldsymbol{n}}
\end{gathered}
$$

Substituting the previous two relations equations (49) and (50) in the velocity and acceleration relations of Eq. (46) gives

$$
\begin{aligned}
& \boldsymbol{a}_{\boldsymbol{n}+1-\alpha_{m}}=\left(1-\alpha_{m}\right) \frac{1}{\beta \Delta t_{s}^{2}}\left[\delta \boldsymbol{q}-\Delta t_{s} \boldsymbol{v}_{\boldsymbol{n}}-\Delta t_{s}^{2}\left(\frac{1}{2}-\beta\right) \boldsymbol{a}_{\boldsymbol{n}}\right] \\
& +\alpha_{m} \boldsymbol{a}_{\boldsymbol{n}} \\
& \boldsymbol{v}_{\boldsymbol{n}+1-\alpha_{f}}=\left(1-\alpha_{f}\right)\left[\boldsymbol{v}_{\boldsymbol{n}}+\Delta t_{s}\left(1-\gamma_{n m}\right) \boldsymbol{a}_{\boldsymbol{n}}+\frac{\gamma_{n m}}{\beta \Delta t_{s}} \boldsymbol{\delta} \boldsymbol{q}-\frac{\gamma_{n m}}{\beta} \boldsymbol{v}_{\boldsymbol{n}}\right. \\
& \left.-\frac{\gamma_{n m} \Delta t_{s}}{\beta}\left(\frac{1}{2}-\beta\right) \boldsymbol{a}_{\boldsymbol{n}}\right]+\alpha_{f} \boldsymbol{v}_{\boldsymbol{n}}
\end{aligned}
$$

Using the tangent stiffness method [39], the internal forces at time step $n+1$ (i.e., $R_{n+1}$ ) can be written as

$$
\begin{gathered}
\boldsymbol{R}_{\boldsymbol{n}+1}=\boldsymbol{R}_{\boldsymbol{n}}+\boldsymbol{K}_{\boldsymbol{t}} \boldsymbol{\delta} \boldsymbol{q} \\
\boldsymbol{R}_{\boldsymbol{n}+1-\alpha_{f}}=\left(1-\alpha_{f}\right)\left(\boldsymbol{R}_{\boldsymbol{n}}+\boldsymbol{K}_{t} \boldsymbol{\delta} \boldsymbol{q}\right)+\alpha_{f} \boldsymbol{R}_{\boldsymbol{n}}
\end{gathered}
$$

Substituting the previous set of equations in the balance equation, Eq. (45) becomes

$$
\boldsymbol{K}_{\mathrm{eff}} \boldsymbol{\delta} \boldsymbol{q}=\boldsymbol{R}_{\boldsymbol{h}}
$$

where $\boldsymbol{K}_{\text {eff }}$ and $\boldsymbol{R}_{\boldsymbol{h}}$ are the effective stiffness matrix and the effective load vector, and they are given as

$$
\boldsymbol{K}_{\mathrm{eff}}=\frac{1-\alpha_{m}}{\beta \Delta t_{s}^{2}} \boldsymbol{M}+\frac{1-\alpha_{f}}{\beta \Delta t_{s}} \boldsymbol{C}+\left(1-\alpha_{f}\right) \boldsymbol{K}_{\boldsymbol{t}}
$$

$$
\begin{aligned}
\boldsymbol{R}_{\boldsymbol{h}} & =\frac{1-\alpha_{m}}{\beta \Delta t_{s}} \boldsymbol{M} \boldsymbol{v}_{\boldsymbol{n}}+\frac{1-\alpha_{m}}{\beta}\left(\frac{1}{2}-\beta\right) \boldsymbol{M} \boldsymbol{a}_{\boldsymbol{n}}-\alpha_{m} \boldsymbol{M} \boldsymbol{a}_{\boldsymbol{n}} \\
& -\boldsymbol{C}\left(1-\alpha_{f}\right) \boldsymbol{V}_{\boldsymbol{n}}-\cdots-\boldsymbol{C}\left(1-\alpha_{f}\right) \Delta t_{s}\left(1-\gamma_{n m}\right) \boldsymbol{a}_{\boldsymbol{n}} \\
& +\boldsymbol{C}\left(1-\alpha_{f}\right) \frac{\gamma_{n m}}{\beta} \boldsymbol{v}_{\boldsymbol{n}}+\boldsymbol{C}\left(1-\alpha_{f}\right) \frac{\gamma_{n m} \Delta t_{s}}{\beta}\left(\frac{1}{2}-\beta\right) \boldsymbol{a}_{\boldsymbol{n}} \\
& -\boldsymbol{C} \alpha_{f} \boldsymbol{v}_{\boldsymbol{n}}-\left(1-\alpha_{f}\right) \boldsymbol{R}_{\boldsymbol{n}}-\alpha_{f} \boldsymbol{R}_{\boldsymbol{n}}+\left(1-\alpha_{f}\right) \boldsymbol{F}_{\boldsymbol{n}+1}+\alpha_{f} \boldsymbol{F}_{\boldsymbol{n}}
\end{aligned}
$$

A step-by-step solution procedure to solve the system of equations using the quantities computed in Eqs. (56) and (57) is given as follows:

1) Initialize $\boldsymbol{q}_{\boldsymbol{o}}$ and its time derivatives.

2) Select a time-step size $\Delta t_{s}$ and a spectral radius parameter $S_{r}$ $\left(0 \leq S_{r} \leq 1\right)$ : this parameter is inversely proportional to the highfrequency dissipation. The time-integration method used in this paper is implicit, and so the choice of time step should be based on the desired solution accuracy and on the stability of the NewtonRaphson algorithm. In the case of flapping-wing simulations, as presented in this work, the time step was also chosen based on the frequency and amplitude of prescribed motion, so the incremental displacements and rotations within a time step (effectively a load step) are small. For example, smaller time steps were needed for larger (i.e., more nonlinear) amplitudes in order to force the NewtonRaphson loop to converge.

3) Compute parameters $\alpha_{f}=-S_{r} /\left(1+S_{r}\right)$ and $\alpha_{m}=\left(1-2 S_{r}\right) /$ $\left(1+S_{r}\right)$.

4) Compute parameters $\gamma_{n m}=0.5+\alpha_{m}-\alpha_{f} \quad$ and $\beta=0.25\left(1+\alpha_{m}-\alpha_{f}\right)^{2}$.

5) Form the effective stiffness matrix from the individual mass, damping, and tangent stiffness matrices using Eq. (56).

6) Form the effective load vector equation (57).

7) Solve for the displacement increments using Eq. (55). To improve the solution accuracy and to avoid the development of numerical instabilities, it is generally necessary to employ iterations within each time step in order to maintain equilibrium [39]. The following are the steps to be followed in a typical iteration $j$ within the iterative loop.

1) Evaluate the $(j-1)$ th approximation to the acceleration, velocities, and displacements using

$$
\begin{gathered}
a_{c o}=\frac{1-\alpha_{m}}{\beta \Delta t_{s}^{2}}, \quad a_{c 1}=\frac{\gamma_{n m}}{\beta \Delta t_{s}} a_{c 2}=\frac{1}{\beta \Delta t_{s}}, \quad a_{c 3}=\frac{1}{2 \beta}-1 \\
a_{c 4}=\frac{\gamma_{n m}}{\beta}-1, \quad a_{c 5}=\frac{\Delta t_{s}}{2}\left(\frac{\gamma_{n m}}{\beta}-2\right) \\
\boldsymbol{a}_{\boldsymbol{n}+1}^{j-1}=a_{c o} \boldsymbol{\delta} \boldsymbol{q}^{j-1}-a_{c 2} \dot{\boldsymbol{q}}_{\boldsymbol{n}}-a_{c 3} \ddot{\boldsymbol{q}}_{n} \\
\boldsymbol{v}_{\boldsymbol{n}+1}^{j-1}=a_{c 1} \boldsymbol{\delta} \boldsymbol{q}^{j-1}-a_{c 4} \dot{\boldsymbol{q}}_{\boldsymbol{n}}-a_{c 5} \ddot{\boldsymbol{q}}_{\boldsymbol{n}}, \quad \boldsymbol{q}_{\boldsymbol{n}+1}^{j-1}=\boldsymbol{q}_{\boldsymbol{n}}+\delta \boldsymbol{q}^{j-1}
\end{gathered}
$$

2) Update nodal rotation matrices using the new approximation to the solution $\boldsymbol{q}$ [last of Eqs. (20) and (59)]. 


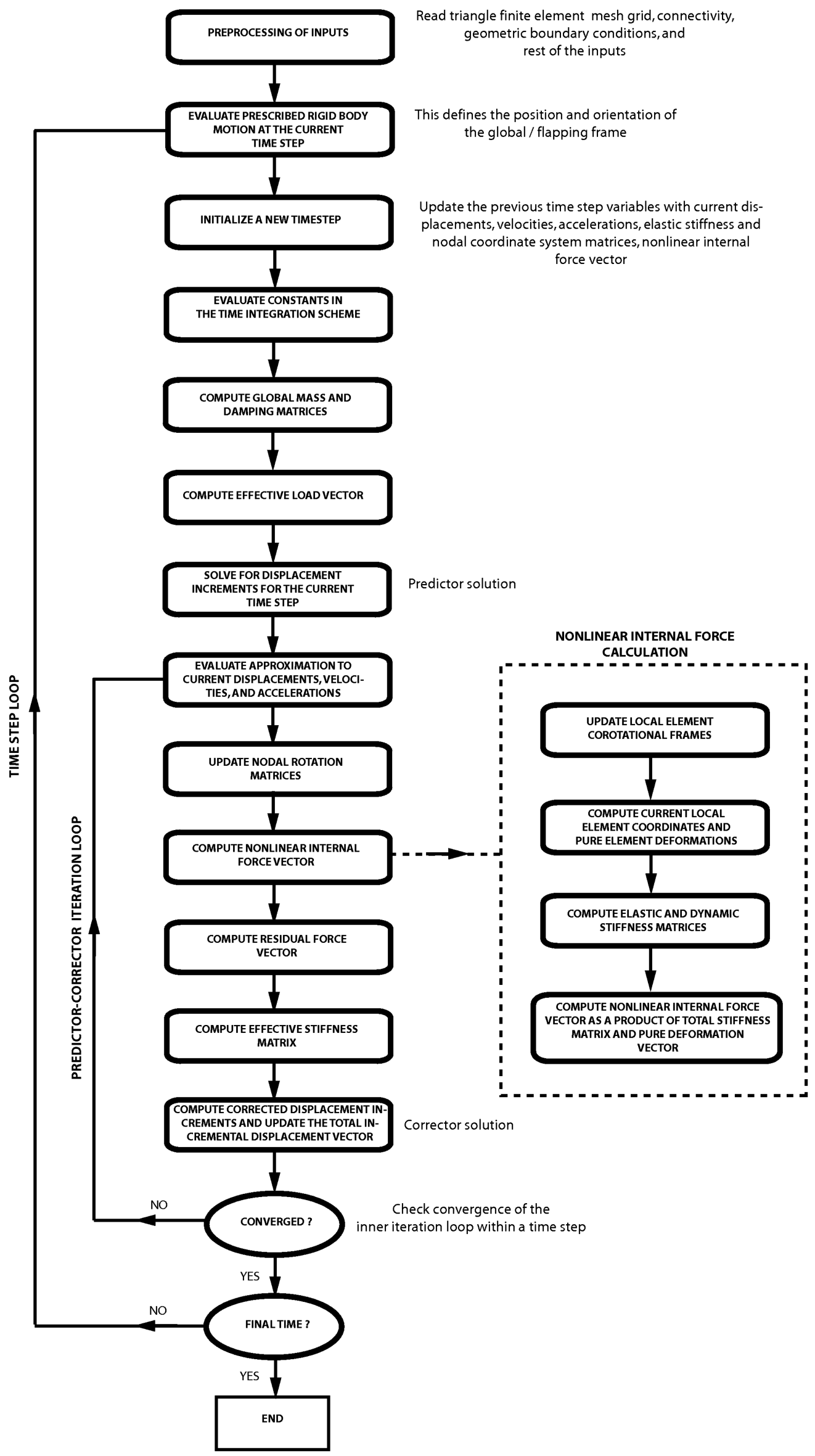

Fig. 2 Nonlinear FE solution process for flapping-wing shell structures implemented in UM/NLAMS. 


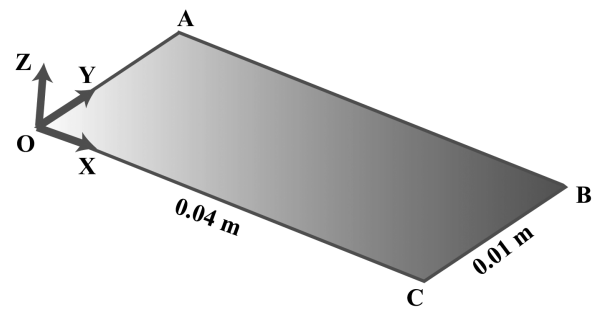

Fig. 3 Rectangular plate configuration used to check rigid body kinematics implementation in UM/NLAMS and MSC.Marc.
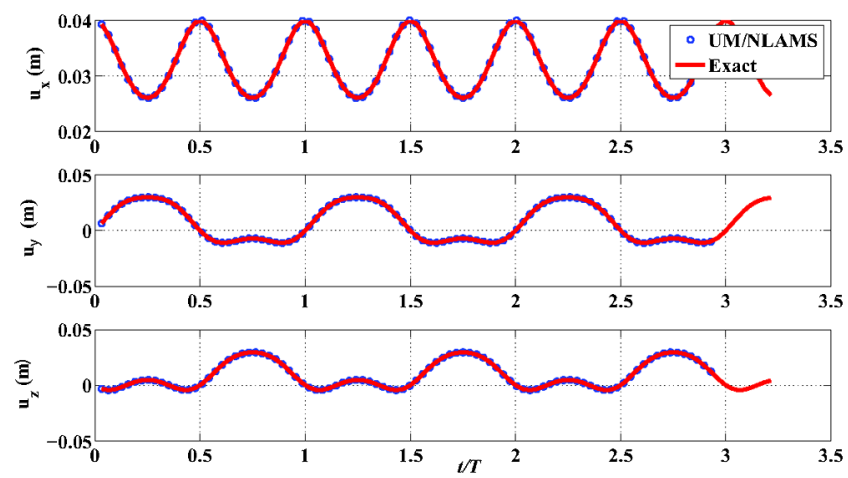

Fig. 4 Displacements extracted at point $B$ in Fig. 3 based on rigid body kinematics prescribed at point $A$ in the same figure.
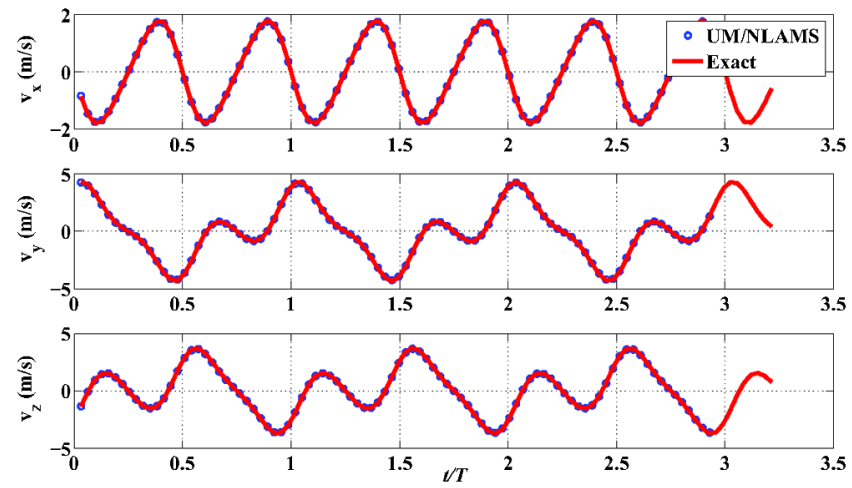

Fig. 5 Translational velocities extracted at point $B$ in Fig. 3 based on rigid body kinematics prescribed at point $\mathrm{A}$ in the same figure.

3) Evaluate the $(j-1)$ th residual force with

$$
\begin{aligned}
& \boldsymbol{R} \boldsymbol{f}_{\boldsymbol{n}+1}^{j-1}=\left(1-\alpha_{f}\right) \boldsymbol{F}\left(\boldsymbol{t}_{\boldsymbol{n}+1}\right)+\alpha_{f} \boldsymbol{F}\left(\boldsymbol{t}_{\boldsymbol{n}}\right)-\boldsymbol{M}\left(1-\alpha_{m}\right) \boldsymbol{a}_{\boldsymbol{n}+1}^{j-1} \\
& \quad-\boldsymbol{M} \alpha_{m} \boldsymbol{a}_{\boldsymbol{n}}-\cdots \boldsymbol{C}\left(1-\alpha_{f}\right) \boldsymbol{v}_{\boldsymbol{n}+1}^{j-1}-\boldsymbol{C} \alpha_{f} \boldsymbol{v}_{\boldsymbol{n}}-\left(1-\alpha_{f}\right) \boldsymbol{R}_{\boldsymbol{n}+1}^{j-1} \\
& \quad-\alpha_{f} \boldsymbol{R}_{\boldsymbol{n}}
\end{aligned}
$$

4) Solve for the $j$ th corrected displacements using

$$
\boldsymbol{K}_{\mathrm{eff}} \boldsymbol{\Delta} \boldsymbol{q}^{j}=\boldsymbol{R} \boldsymbol{f}_{n+1}^{j-1}
$$

5) Evaluate the corrected displacement increments with

$$
\delta \boldsymbol{q}^{j}=\delta \boldsymbol{q}^{j-1}+\Delta \boldsymbol{q}^{j}
$$

6) Check for convergence of the iteration,

$$
\frac{\left|\Delta q^{j}\right|}{\left|\boldsymbol{q}_{t}+\delta q^{j}\right|} \leq \text { tol }
$$

where tol is convergence tolerance for the iteration.

7) If the solution is not converged, return to the first step; if it does, proceed to the next time step.

For a specific choice of the parameters involved in the generalized$\alpha$ method, other integration schemes could be obtained. For example, if $\alpha_{f}=0$ and $\alpha_{m}=0$, the method reduces to the standard Newmark scheme. The primary goal of the generalized- $\alpha$ method is to provide the user with control over high-frequency dissipation while limiting the impact on the low-frequency dynamics. In flapping-wing aeroelastic simulations, this method could prove to be very beneficial in dissipating nonphysical high-frequency oscillations that result due to poor spatial resolution.

The generalized- $\alpha$ method was extended to systems of first-order differential equations by Jansen et al. [40] and later applied by Shearer and Cesnik [24] in the flight dynamic analysis of a highly flexible aircraft.

Figure 2 highlights the key steps involved in the solution process of UM/NLAMS.

\section{Verification and Validation Studies of University of Michigan Nonlinear Membrane Shell Solver}

\section{A. Rigid Body Kinematics}

As a check for the rigid body kinematics implementation in the solver UM/NLAMS, a simple test was conducted on a rigid rectangular plate, shown in Fig. 3 , actuated at point A with prescribed large amplitude sinusoidal rotation functions in three dimensions (all in phase). The amplitudes of rotation are 30,45 , and $80 \mathrm{deg}$ about $X$ (along span), $Y$ (along chord), and $Z$ (vertical) directions, respectively. Three-dimensional displacements and velocities were extracted at an arbitrary point B (also shown in Fig. 3) and are plotted in Figs. 4 and 5. The results from UM/NLAMS have an excellent correlation with the exact solution computed analytically using the rotation tensor corresponding to the rotation vector [34].

\section{B. Nonlinear Static Structural Response}

Three cantilever plate configurations subjected to different static loads (previously studied in $[\underline{4}, \underline{41}]$ ) are considered in this subsection. Results presented include tip displacement as a function of the applied load in all the cases discussed.

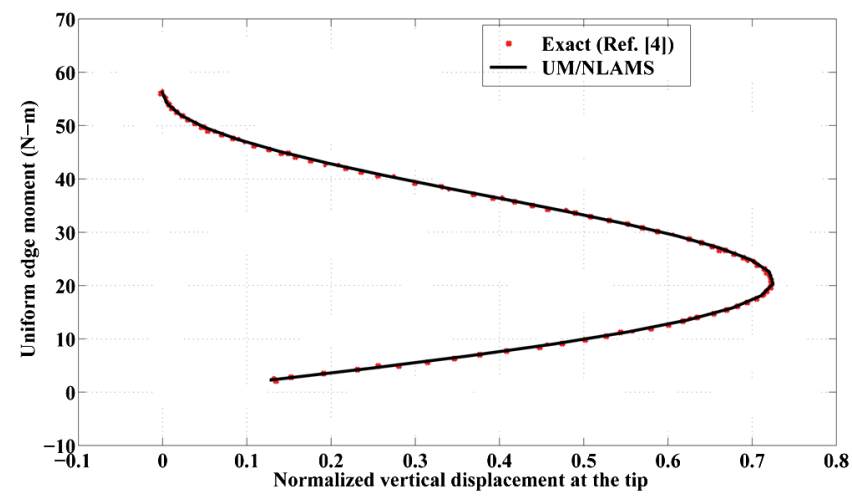

Fig. 6 Normalized tip displacement as a function of the applied moment for the plate in case 1 (displacement is normalized with respect to plate length). 


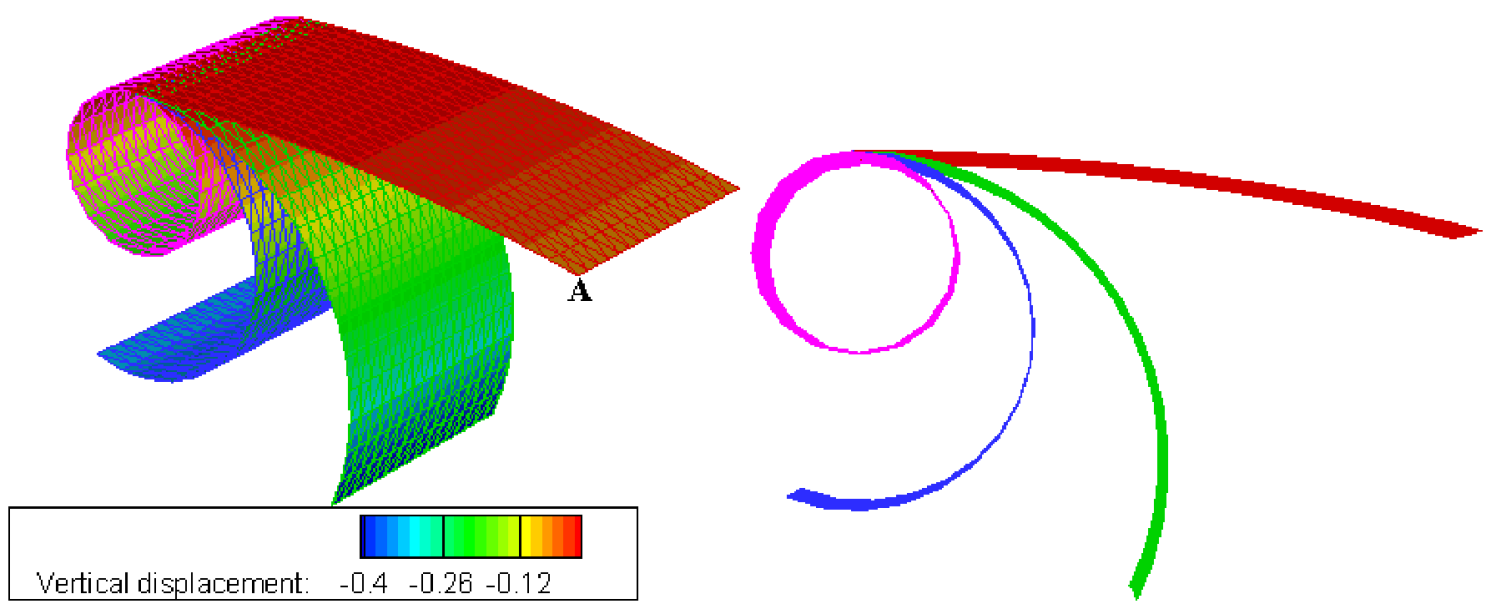

Fig. 7 Snapshots of static wing deformation for the plate in case 1 (legend in meters).

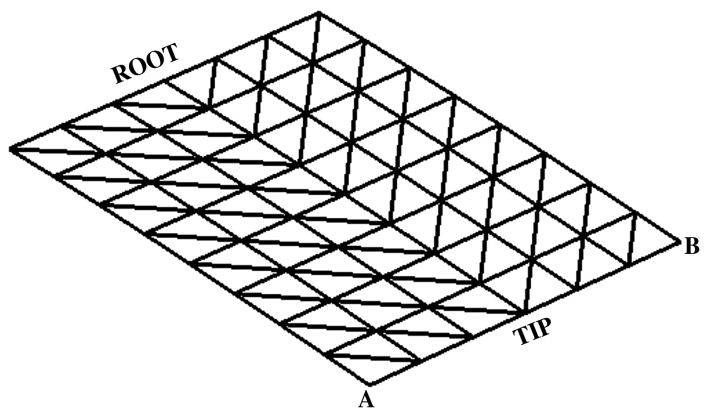

Fig. 8 FE mesh configuration for the plate in case 2.

\section{Case 1: Cantilever Plate Subjected to Uniform End Moments}

This case is used as one of the reference test cases to evaluate the geometrically nonlinear static capability of UM/NLAMS. It corresponds to a cantilevered isotropic plate of aspect ratio two subjected to uniform applied moments along the tip edge. The key parameters related to this case are included in Table 1 . The analytical solution for the case is given in [4] and is used for comparison with results obtained from UM/NLAM $S$. Figure $\underline{6}$ shows the normalized vertical displacement at a point on the tip (point A in Fig. 7) versus the applied moment. The displacement is normalized with respect to the length of the plate (also for all the cases discussed next unless specified otherwise). The plot compares the solution computed in UM/NLAMS with those provided in [4] (which also discusses validation with analytical solutions). As seen in Fig. 6, there is an excellent match between the two results. The maximum tip deflection obtained in this case is $0.4 \mathrm{~m}$, which corresponds to $73 \%$ of the plate length. Because of the applied moment, the cantilever plate forms a circular arc, as shown in Fig. 7. To compute the solution as a function of the applied load, a load control approach was followed in this work: the maximum load in the analysis was broken into several load steps and applied in increments. For each load increment, nonlinear static equilibrium was sought to compute the corresponding static response. The convergence criterion within each load step loop was

Table 2 Parameters associated with case 2

\begin{tabular}{lc}
\hline \hline Parameter & Value \\
\hline Plate length & $40 \mathrm{~m}$ \\
Plate width & $30 \mathrm{~m}$ \\
Plate thickness & $0.4 \mathrm{~m}$ \\
Young's modulus & $0.12 \mathrm{GPa}$ \\
Poisson's ratio & 0.3 \\
Number of FEs & 96 \\
Number of load steps & 25 \\
Error limit & $10^{-4}$ \\
\hline \hline
\end{tabular}

chosen as the absolute difference in the Euclidean norm of the entire solution vector computed in any two consecutive Newton-Raphson iterations. For the current case, the tolerance for it was set to $10^{-3}$.

\section{Case 2: Cantilever Plate Subjected to an End Lateral Load}

This case corresponds to an isotropic cantilever plate subjected to a lateral load at one of its free corners (node A of Fig. $\underline{8}$ ). The key parameters related to this case are included in Table 2. The results obtained from UM/NLAMS are compared with those published in [41]. Figure 9 shows the normalized vertical displacement at the tip (node B of Fig. $\underline{8}$ ) versus the applied load. As before, the displacement is normalized with respect to the length of the plate. The maximum displacement found in this case was $45 \%$ of the plate length. Figure 10 shows snapshots of the static wing deformation for three different load steps. The maximum difference in the static displacement computed in UM/NLAMS and that found in [41] is up to $1 \%$ of the plate length. A reason for this is the difference in the solution formulation of UM/NLAMS and that of [41]. While the former is based on the CR form of the TL method, the latter is that of the UL method.

\section{Case 3: Cantilever Plate Subjected to an End Lateral Load}

This case corresponds to an isotropic cantilever plate subjected to shear forces at the three nodes of the tip (nodes A, B, and C in Fig. 11). The load at nodes A and C is $10 \mathrm{~N}$, whereas at node $\mathrm{B}$, it is $20 \overline{\mathrm{N}}$. Similar to the previous two cases, the load is applied incrementally in several load steps. The key parameters related to this case are included in Table 3. The solution obtained from UM/ NLAMS in this case is compared with the analytical solution published in [4]. Figure 12 shows the normalized vertical displacement at the tip (node $\overline{\mathrm{A}}$ in Fig. 11) versus the total applied load (sum of the loads on points $\mathrm{A}, \overline{\mathrm{B}}$, and $\mathrm{C}$ ). The maximum

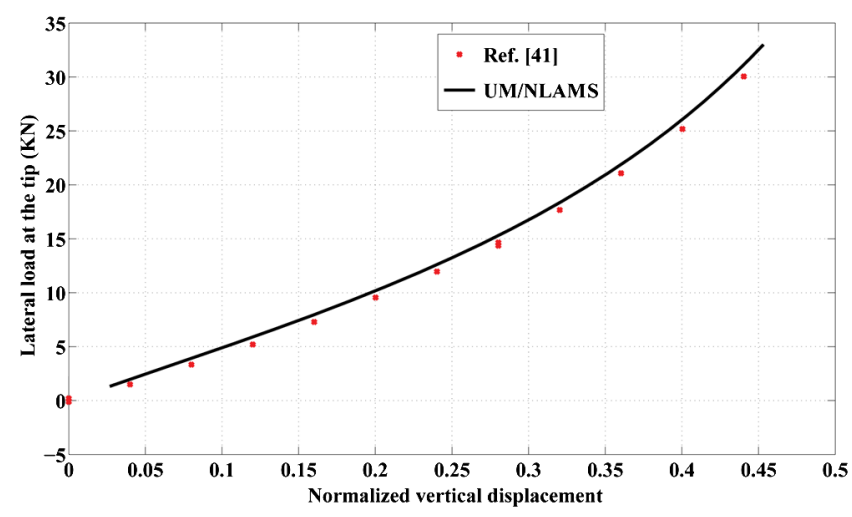

Fig. 9 Normalized tip displacement as a function of applied load for the plate in case 2 (displacement is normalized with respect to plate length). 

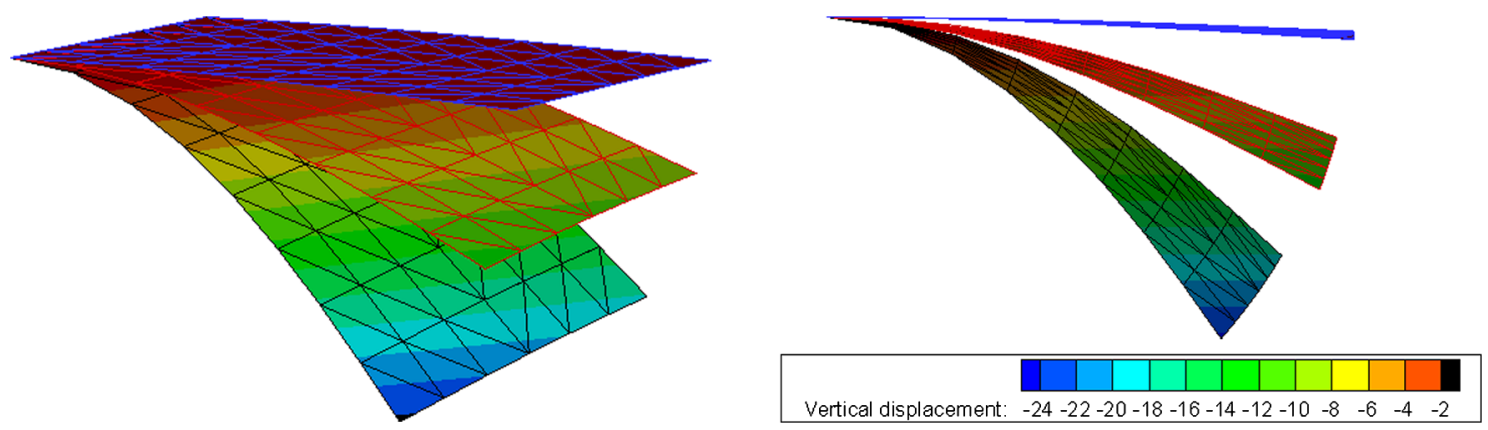

Fig. 10 Snapshots of wing deformation for the plate in case 2 (legend in meters).

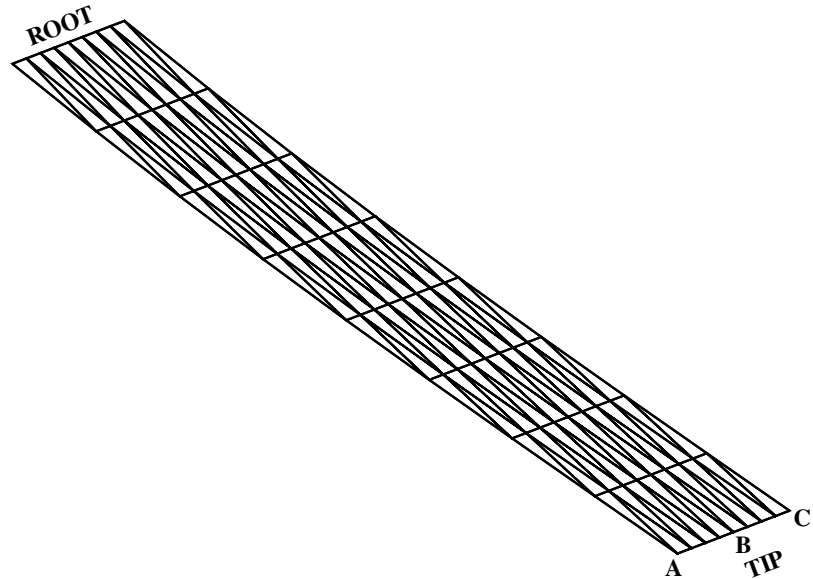

Fig. 11 FE mesh configuration for the plate in case 3.

displacement found in this case was approximately $68 \%$ of the plate length. The agreement is very good up to $60 \%$ deformation, after which UM/NLAMS struggles to converge within the maximum number of subiterations (200) within a load step. This presents itself as a softer behavior than that of [4]. The cause for this behavior is unknown and requires further investigation.

\section{Dynamic Structural Response}

In this subsection, both rectangular and elliptic plate configurations are actuated with different types of prescribed dynamic motions. In all the cases considered, responses computed using UM/ NLAMS are compared with either those from MSC.Marc or with available experimental data. Results presented include tip displacement as a function of time and snapshots of three-dimensional wing deformation.

\section{Case 4: Rectangular Plate Prescribed with Single-Degree-of-Freedom} Flap Rotation

A rectangular aluminum plate shown in Fig. 13 was prescribed with a single-DOF large amplitude flap rotation about an axis running through the chord. In the figure, the square block at the wing

Table 3 Parameters associated with case 3

\begin{tabular}{lc}
\hline \hline Parameter & Value \\
\hline Plate length & $0.1 \mathrm{~m}$ \\
Plate width & $0.01 \mathrm{~m}$ \\
Plate thickness & $0.001 \mathrm{~m}$ \\
Young's modulus & $117.72 \mathrm{GPa}$ \\
Poisson's ratio & 0. \\
Number of FEs & 128 \\
Number of load steps & 25 \\
Error limit & $10^{-4}$ \\
\hline \hline
\end{tabular}

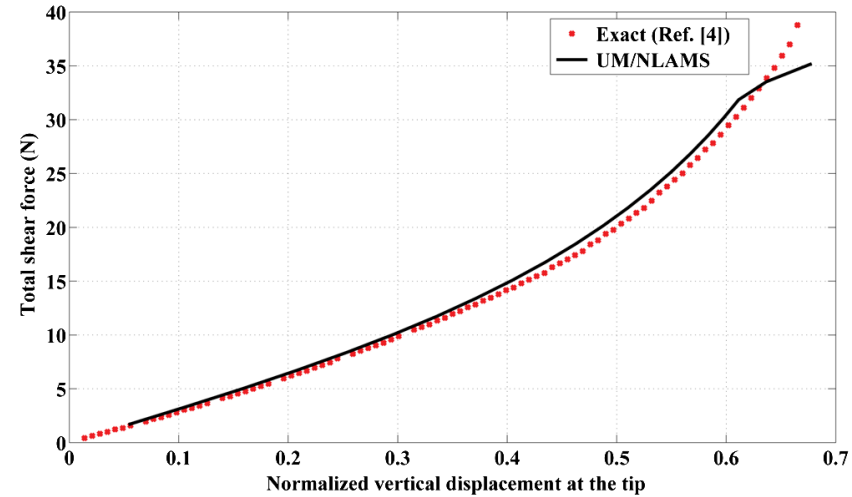

Fig. 12 Normalized tip displacement as a function of the magnitude of the total applied load for the plate in case 3 (displacement is normalized with respect to plate length).

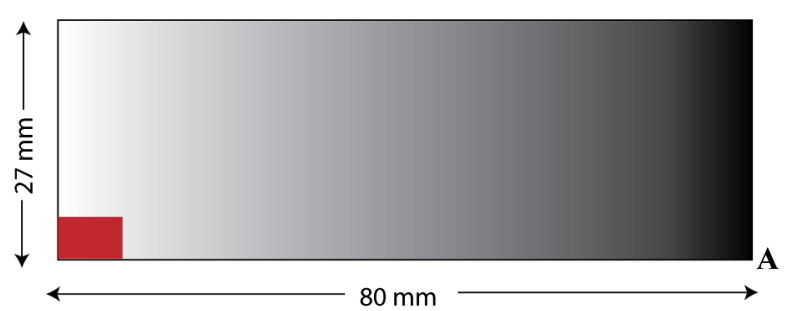

Red color indicates the region ( $5 \mathrm{~mm} \times 5 \mathrm{~mm}$ ) on the wing that is in contact with the flapping mechanism

Fig. 13 Rectangular flat plate flapping-wing configuration for case 4.

root is constrained in all DOFs with respect to the global frame, and the rotation was prescribed as a sinusoidal variation. This enabled the simulation to start from zero initial displacement and velocity, obviating the need for a special starting procedure, as would have been the case if a sine variation was prescribed. The key parameters

Table 4 Parameters associated with case 4

\begin{tabular}{lc}
\hline \hline Parameter & Value \\
\hline Plate length & $80 \mathrm{~mm}$ \\
Plate width & $27 \mathrm{~mm}$ \\
Plate thickness & $0.2 \mathrm{~mm}$ \\
Young's modulus & $70 \mathrm{GPa}$ \\
Poisson's ratio & 0.3 \\
Material density & $2700 \mathrm{~kg} / \mathrm{m}^{3}$ \\
Prescribed flap rotation profile & $1-$ cosine \\
Flapping frequency & 5,10, and $30 \mathrm{~Hz}$ \\
Flapping amplitude & $17 \mathrm{deg}$ \\
Time-step sizes & $1.5 \times 10^{-4}$ and $10^{-5} \mathrm{~s}$ \\
Number of FEs & 512 \\
Error limit & $10^{-4}$ \\
\hline \hline
\end{tabular}




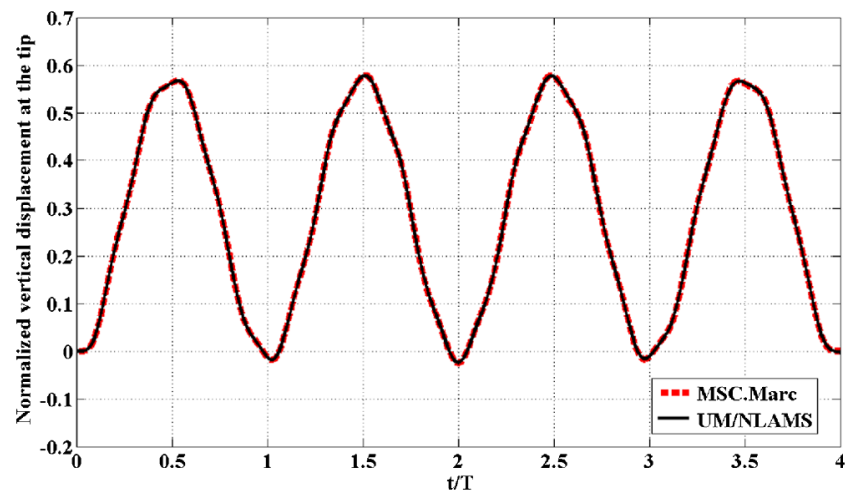

Fig. 14 Rectangular flat plate response due to flapping excitation $(5 \mathrm{~Hz})$ in case 4 (displacement is normalized with respect to plate length).

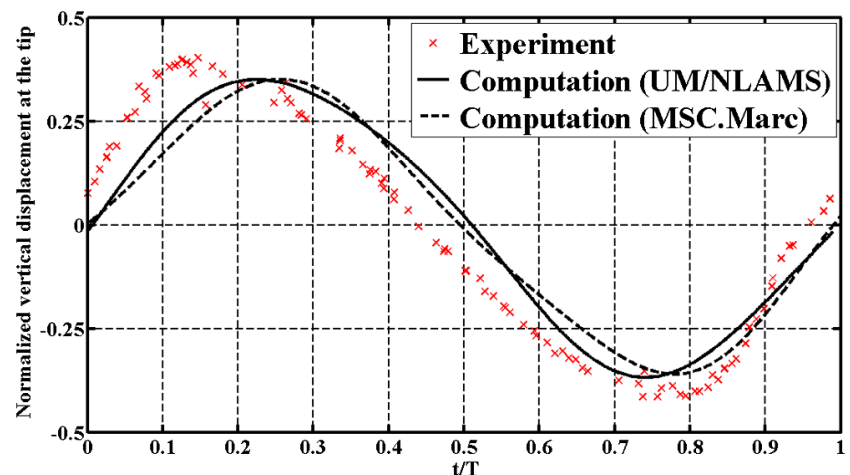

Fig. 15 Rectangular flat plate response due to flapping excitation $(10 \mathrm{~Hz})$ in case 4 (displacement is normalized with respect to plate length).

related to this case are included in Table 4. Figures 14-16 show the normalized vertical displacement at the tip (point A in Fig. 13) as a function of nondimensional time (time normalized with respect to the period of the flap rotation) for three different flapping frequencies: 5 , 10 , and $30 \mathrm{~Hz}$, respectively. The results are compared with either those obtained from the commercial FE solver MSC.Marc or with available experimental data [42]. The bilinear thin-triangular shell element number 138 is used to discretize the wing in MSC.Marc (also for all the MSC.Marc cases discussed here). The time-integration schemes used are the nondissipative form of the Newmark and the generalized- $\alpha$ methods. The former was used in the 5 and $10 \mathrm{~Hz}$ cases, whereas the latter was used in the $30 \mathrm{~Hz}$ case with a spectral radius value set to 0.4 . The time-step size used in the 5 and $10 \mathrm{~Hz}$ cases was $1.5 \times 10^{-4} \mathrm{~s}$ and, in the $30 \mathrm{~Hz}$ case, it was $10^{-5} \mathrm{~s}$. The convergence criterion for the Newton-Raphson convergence loop is

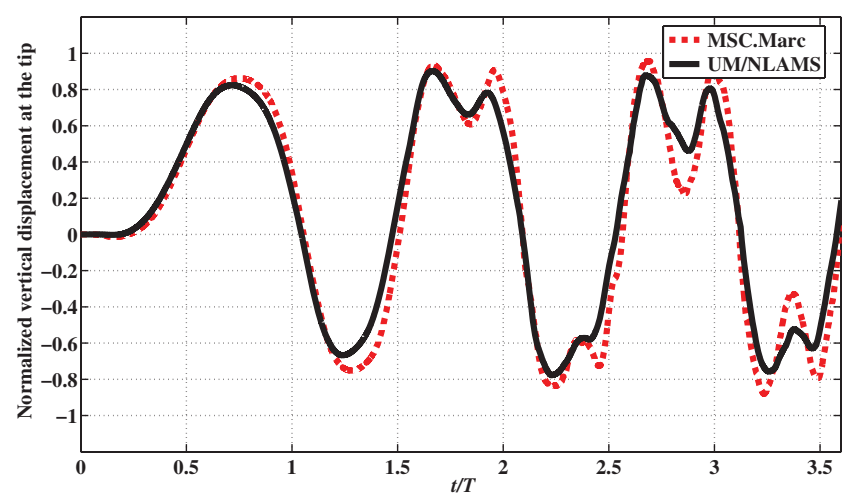

Fig. 16 Rectangular flat plate response due to flapping excitation $(30 \mathrm{~Hz})$ in case 4 (displacement is normalized with respect to plate length).
Table 5 Parameters associated with case 5

\begin{tabular}{lc}
\hline \hline Parameter & Value \\
\hline Plate length & $0.3 \mathrm{~m}$ \\
Plate width & $0.1 \mathrm{~m}$ \\
Plate thickness & $0.001 \mathrm{~m}$ \\
Young's modulus & $210 \mathrm{GPa}$ \\
Poisson's ratio & 0.3 \\
Material density & $7800 \mathrm{~kg} / \mathrm{m}^{3}$ \\
Prescribed plunge profile & $1-$ cosine \\
Plunge frequency & $1.78 \mathrm{~Hz}$ \\
Plunge amplitude & $0.0175 \mathrm{~m}$ \\
Time-step size & $10^{-3} \mathrm{~s}$ \\
Number of FEs & 1150 \\
Error limit & $10^{-4}$ \\
\hline
\end{tabular}

a check on the absolute difference in the Euclidean norm of the entire solution vector computed in any two consecutive iterations: set to $10^{-4}$ in the three cases considered here. As seen from the results in Fig. 14, the agreement between UM/NLAMS and MSC.Marc for the $5 \mathrm{~Hz}$ frequency is very good. With an increase in frequency $(30 \mathrm{~Hz})$, differences start to become apparent (Fig. 16), which is presumably due to the stronger geometric nonlinearities as well as the increased effect of the transient terms in Eq. (42). While the exact cause for the discrepancies will require further investigation, part of the reason could be the fact that MSC.Marc does not use the CR approach. In any case, future studies should be conducted to address which solution is more accurate under higher frequency regimes. Also, as seen in Fig. 15, while the agreement between UM/NLAMS, MSC.Marc, and the experiment is reasonable in amplitude, there is some disagreement in phase. Each computational time step (particularly including assembling of matrices and solving the system of equations) for this case needed $20 \mathrm{~s}$ (approximately) of CPU time on a single-node Intel Pentium 4 CPU $3.4 \mathrm{GHz}$ processor.

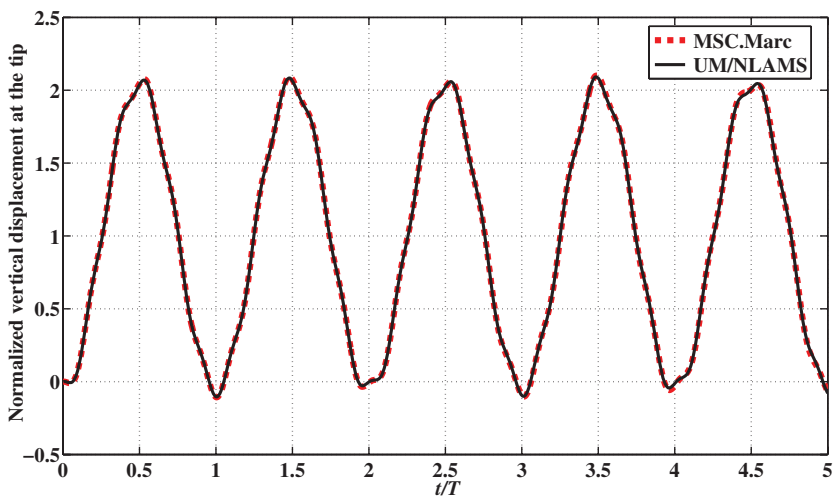

Fig. 17 Rectangular plate response due to plunge excitation in case 5 (displacement is normalized with respect to the amplitude of plunge).

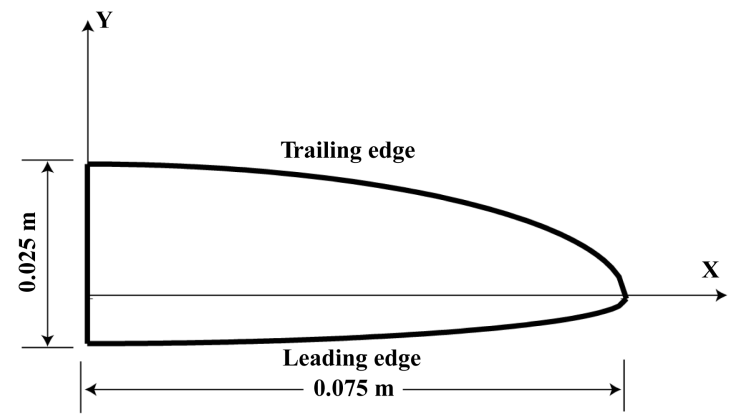

Fig. 18 Zimmerman elliptic plate flapping-wing configuration. 
Table 6 Parameters associated with case 6

\begin{tabular}{lc}
\hline \hline Parameter & Value \\
\hline Plate length at quarter chord & $0.075 \mathrm{~m}$ \\
Plate width at the root & $0.025 \mathrm{~m}$ \\
Plate thickness & $0.2 \times 10^{-3} \mathrm{~m}$ \\
Young's modulus & $7.34 \mathrm{GPa}$ \\
Poisson's ratio & 0.3 \\
Material density & $1740 \mathrm{~kg} / \mathrm{m}^{3}$ \\
Prescribed rotation profile & $1-\operatorname{cosine}$ \\
Flap frequency & $10 \mathrm{~Hz}$ \\
Flap amplitudes & 5,15, and $5 \mathrm{deg}$ about \\
& $x, y$, and $z$ axes, respectively \\
Time-step size & $1.5 \times 10^{-4} \mathrm{~s}$ \\
Number of FEs & 244 \\
Error limit & $10^{-4}$ \\
\hline \hline
\end{tabular}

2. Case 5: Rectangular Cantilever Plate Prescribed with Pure Plunge Motion

A rectangular cantilever steel plate was prescribed with a pure plunge motion at the root. The key parameters related to this case are included in Table 5. Figure 17 shows the normalized vertical displacement at the tip versus the normalized time, with a comparison between UM/NLAMS and the commercial FE solver MSC.Marc. The displacements and time are normalized with respect to the plunge amplitude and the period of plunge, respectively. The timeintegration method used is the Newmark scheme. As seen in Fig. 17, there is an excellent match between the results computed in both the codes. Each computational time step (particularly including assembling of matrices and solving the system of equations) for this case needed $10 \mathrm{~s}$ (approximately) of CPU time on a single-node Intel Pentium 4 CPU $3.4 \mathrm{GHz}$ processor.

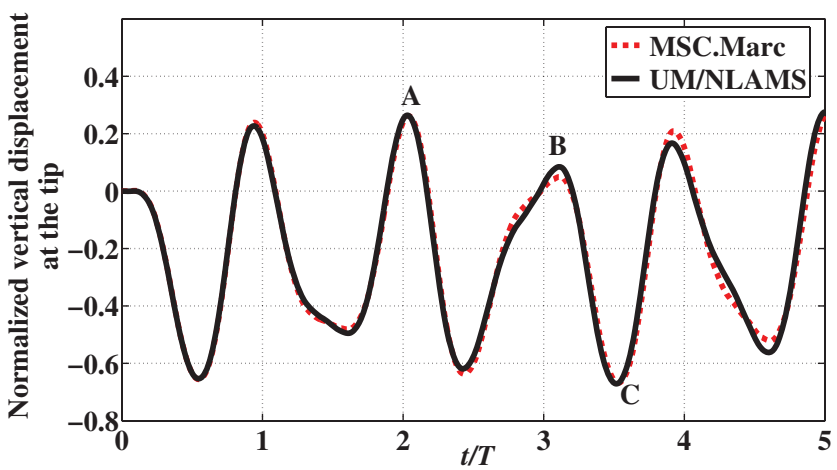

Fig. 19 Elliptic flat plate response to prescribed flap rotations in case 6 (displacement is normalized with respect to plate length).

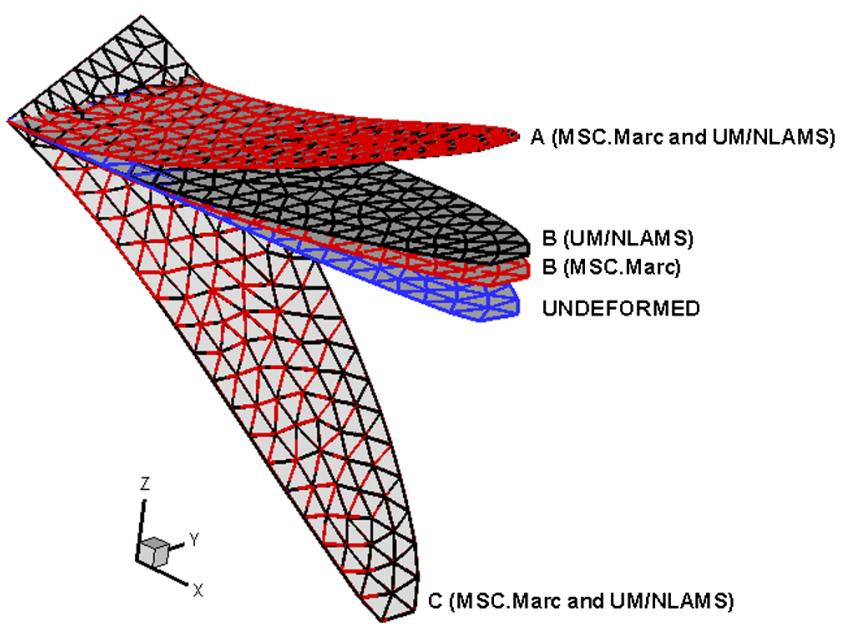

Fig. 20 Snapshot of dynamic wing deformation in case 6 (perspective).

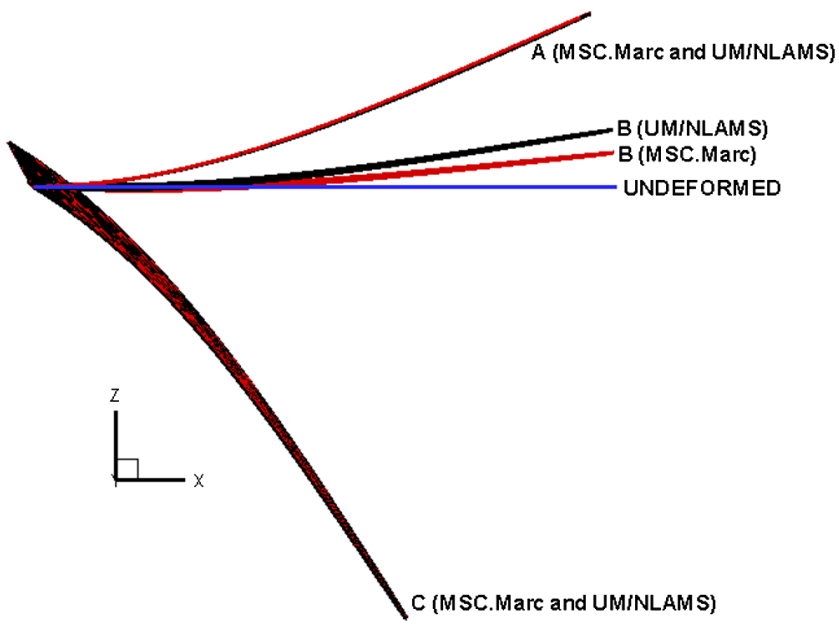

Fig. 21 Snapshot of dynamic wing deformation in case 6 (side view).

3. Case 6: Elliptic Cantilever Plate Prescribed with Rotations About all Axes

An elliptic isotropic plate of the Zimmerman planform (shown in Fig. 18) is cantilevered along the root with respect to the global frame and is actuated at its leading-edge point on the root, with prescribed rotation functions about all three coordinate axes. The Zimmerman planform is simply formed by two ellipses intersecting at the quarterchord point. Mathematically, it is defined by

$$
\begin{aligned}
& \left(\frac{x}{R}\right)^{2}+\left(\frac{y}{c_{1}}\right)^{2}=1\left\{\begin{array}{l}
0 \leq x \leq R \\
0 \leq y \leq c_{1}
\end{array}\right. \\
& \left(\frac{x}{R}\right)^{2}+\left(\frac{y}{c_{2}}\right)^{2}=1\left\{\begin{array}{l}
0 \leq x \leq R \\
-c_{2} \leq y \leq 0
\end{array}\right.
\end{aligned}
$$

where

$$
\begin{gathered}
c_{1}=0.75 c, \quad c_{2}=0.75 c, \quad S=\frac{c_{1} R \pi}{2}+\frac{c_{2} R \pi}{2}=\frac{c R \pi}{2} \\
A_{r}=\frac{4 R^{2}}{S}=\frac{8 R}{c \pi}
\end{gathered}
$$

In Eq. (65), $c$ is the chord length at the root, $R$ is the length of the wing defined as the length of the quarter-chord line along the wingspan, and $A_{r}$ is the aspect ratio of the full wing, which is 7.65 in this case. The key parameters related to this case are included in Table 6 . Figure 19 shows the normalized vertical displacement at the tip (quarter-chord point) versus the normalized time, with a comparison between UM/NLAMS and MSC.Marc. As seen from the figure, overall, there is a very good correlation between them, barring some discrepancies at specific time instants. Figures 20 and 21 show snapshots of wing deformation computed in both $\overline{\mathrm{UM}} / \mathrm{NL} \overline{\mathrm{AM}} \mathrm{S}$ and MSC.Marc for three different time instants (A, B, and C of Fig. 19). As seen from the snapshots, there is an excellent agreement between the three-dimensional wing deformation computed in UM/NLAMS and MSC.Marc at points A and C, but there is a slight discrepancy (approximately $3 \%$ of plate length) at point $\mathrm{B}$, which is also seen from Fig. 19.

\section{Conclusions}

The development of a nonlinear structural dynamics solver suitable for analyzing plate/shell-like flapping-wing structures that is based on the CR form of the TL approach was presented. The formulation of the present element is based on the combination of a DKT plate bending element and an OPT membrane element and included a consistent mass and dynamic stiffness/damping matrices to analyze the structural dynamics of a flapping wing. Numerical investigations related to partial verification and validation of the solver are presented. Results showed good agreement between the 
solutions obtained using this element with available analytical/ experimental/commercial solutions. While there are several commercial nonlinear FE solvers available, the use of the CR approach that uses existing linear FEs in a nonlinear context resulting in a simplified FE formulation is rare. Furthermore, typical numerical examples presented in this work to illustrate the structural dynamics response of flapping wings will be useful to the flapping-wing community at large.

\section{Acknowledgments}

This work was supported by the U.S. Air Force Office of Scientific Research Multidisciplinary University Research Initiative grant and by the Michigan/U. S. Air Force Research Laboratory/Boeing Collaborative Center in Aeronautical Sciences.

\section{References}

[1] S. Combes, S., and Daniel, T., "Flexural Stiffness in Insect Wings I.Scaling and the Influence of Wing Venation," Journal of Experimental Biology, Vol. 206, No. 17, 2003, pp. 2979-2987. doi: $10.1242 /$ jeb. 00523

[2] Wootton, J., "Support and Deformability in Insect Wings," Journal of Zoology, Vol. 193, No. 4, 1981, pp. 447-468. doi:10.1111/j.1469-7998.1981.tb01497.x

[3] Wasfy, T., "Computational Strategies for Flexible Multibody Systems," Applied Mechanics Reviews, Vol. 56, No. 6, 2003, pp. 553-613. doi:10.1115/1.1590354

[4] Khosravi, P., R. Ganesan, R., and Sedaghati, R., "Co-Rotational Nonlinear Analysis of Thin Plates and Shells," International Journal of Numerical Methods in Engineering, Vol. 69, No. 4, 2007, pp. 859-885. doi:10.1002/nme.1791

[5] Khosravi, P., R. Ganesan, R., and Sedaghati, R., "An Efficient Facet Shell Element for Co-Rotational Nonlinear Analysis of Thin and Moderately Thick Laminated Composite Structures," Computers and Structures, Vol. 86, No. 9, 2008, pp. 850-858. doi:10.1016/j.compstruc.2007.04.010

[6] Tsang, T., "Dynamic Analysis of Highly Deformable Bodies Undergoing Large Rotations," Ph.D. Thesis, Univ. of Arizona, Tucson, AZ, 1993.

[7] Battini, J., "A Modified Corotational Framework for Triangular Shell Elements," Computer Methods in Applied Mechanics and Engineering, Vol. 196, Nos. 13-16, 2007, pp. 1905-1914.

doi:10.1016/j.cma.2006.10.006

[8] Behdinan, K., Stylianou, M., and Tabarrok, B., "Co-Rotational Dynamic Analysis of Flexible Beams," Computer Methods in Applied Mechanics and Engineering, Vol. 154, Nos. 3-4, 1998, pp. 151-161. doi:10.1016/S0045-7825(97)00124-2

[9] Elkaranshawy, H., and Dokainish, M., "Co-Rotational Finite Element Analysis of Planar Flexible Multibody Systems," Computers and Structures, Vol. 54, No. 5, 1995, pp. 881-890. doi:10.1016/0045-7949(94)00346-5

[10] Criseld, M., Galvanetto, U., and Jelenic, G., "Dynamics of 3-D Co-Rotational Beams," Computational Mechanics, Vol. 20, No. 6, 1997, pp. 507-519. doi:10.1007/s004660050271

[11] Hsiao, K., and Yang, R., "A Co-Rotational Formulation for Nonlinear Dynamic Analysis of Curved Euler Beam," Computers and Structures, Vol. 54, No. 6, 1995, pp. 1091-1097. doi:10.1016/0045-7949(94)00399-N

[12] Devloo, P., Geradin, M., and Fleury, R., "A Co-Rotational Formulation For the Simulation of Flexible Mechanisms," Multibody System Dynamics, Vol. 4, Nos. 2-3, 2000, pp. 267-295. doi:10.1023/A:1009884131140

[13] Hsiao, K., Lin, J., and Lin, W., "A Consistent Co-Rotational Finite Element Formulation for Geometrically Nonlinear Dynamic Analysis of 3-D Beams," Computer Methods in Applied Mechanics and Engineering, Vol. 169, Nos. 1-2, 1999, pp. 1-18. doi:10.1016/S0045-7825(98)00152-2

[14] Pacoste, C., "Co-Rotational Flat Facet Triangular Elements for Shell Instability Analysis," Computer Methods in Applied Mechanics and Engineering, Vol. 156, Nos. 1-4, 1998, pp. 75-110. doi: 10.1016/S0045-7825(98)80004-2

[15] Zhong, H., and Criseld, M., "An Energy-Conserving Co-Rotational Procedure for the Dynamics of Shell Structures," Engineering Computations, Vol. 15, No. 5, 1998, pp. 552-576. doi: $10.1108 / 02644409810225715$
[16] Wasfy, T., "Modeling Continuum Multibody Systems Using the Finite Element Method and Element Convected Frames, Machine Elements, and Machine Dynamics," 23rd ASME Mechanisms Conference, American Soc. of Mechanical Engineers, Fairfield, NJ, 1994, pp. 327 336.

[17] Relvas, A., and Suleman, A., "Fluid Structure Interaction Modelling of Nonlinear Aeroelastic Structures Using the Finite Element Corotational Theory," Journal of Fluids and Structures, Vol. 22, No. 1, 2006, pp. $59-75$

doi:10.1016/j.jfluidstructs.2005.09.007

[18] Meek, J., and Wang, Y., "Nonlinear Static and Dynamic Analysis of Shell Structures with Finite Rotations," Computer Methods in Applied Mechanics and Engineering, Vol. 162, Nos. 1-4, 1998, pp. 301-315. doi:10.1016/S0045-7825(97)00349-6

[19] Barut, A., Das, M., and Madenci, E., "Nonlinear Deformations of Flapping Wings on a Micro Air Vehicle," 47th AIAA/ASME/ASCE/ AHS/ASC Structures, Structural Dynamics, and Materials Conference, Newport, RI, AIAA Paper 2006-1662, May 2006.

[20] Batoz, J., Bathe, K., and Ho, L., "A Study of Three-Node Triangular Plate Bending Elements," International Journal for Numerical Methods in Engineering, Vol. 15, No. 12, 1980, pp. 1771-1812. doi:10.1002/nme.1620151205

[21] Felippa, C., "A Study of Optimal Membrane Triangles with Drilling Freedoms," Computer Methods in Applied Mechanics and Engineering, Vol. 192, Nos. 16-18, 2003, pp. 2125-2168. doi:10.1016/S0045-7825(03)00253-6

[22] Nour-Omid, B., and Rankin, C., "Finite Rotation Analysis and Consistent Linearization Using Projectors," Computer Methods in Applied Mechanics and Engineering, Vol. 93, No. 3, 1991, pp. 353384

[23] J. Chung, J., and Hulbert, G., "A Time Integration Algorithm for Structural Dynamics With Improved Numerical Dissipation," Journal of Applied Mechanics, Vol. 60, No. 2, June 1993, pp. 371-375. doi: $10.1115 / 1.2900803$

[24] Shearer, C., and Cesnik, C., "Modified Generalized- $\alpha$ Method for Integrating Governing Equations of Very Flexible Aircraft," 47th AIAA/ASME/ASCE/AHS/ASC Structures, Structural Dynamics, and Materials Conference, Newport, RI, AIAA Paper 2006-1747, May 2006.

[25] Bathe, K., Ramm, E., and Wilson, E., "Finite Element Formulations for Large Deformation Dynamic Analysis," International Journal of Numerical Methods in Engineering, Vol. 9, No. 2, 1975, pp. 353-386. doi:10.1002/nme.1620090207

[26] Battini, J., and Pacoste, C., "On the Choice of Local Element Frame for Co-Rotational Triangular Shell Elements," Communications in Numerical Methods in Engineering, Vol. 20, No. 10, 2004, pp. 819825 .

doi: $10.1002 / \mathrm{cnm} .710$

[27] Mattiasson, K., and Samuelson, A., "Total and Updated Lagrangian Forms of the Co-Rotational Finite Element Formulation in Geometrically and Materially Nonlinear Analysis," Numerical Methods for Nonlinear Problems, Univ. Politecnia de Barcelona, Barcelona, April 1984.

[28] Relvas, A., and Suleman, A., "Application of the Co-Rotational Structural Kinematics and Euler Flow to Two Dimensional Nonlinear Aeroelasticity," Computers and Structures, Vol. 85, Nos. 17-18, 2007, pp. 1372-1381. doi:10.1016/i.compstruc. 2006.08.089

[29] Attar, P., and Gordnier, R., "Aeroelastic Prediction of the Limit Cycle Oscillations of a Cropped Delta Wing," Journal of Fluids and Structures, Vol. 22, No. 1, 2006, pp. 45-58 doi:10.1016/j.jfluidstructs.2005.08.010

[30] de Veubeke, B., "The Dynamics of Flexible Bodies," International Journal of Engineering Science, Vol. 14, No. 10, 1976, pp. 895-913. doi:10.1016/0020-7225(76)90102-6

[31] Nygard, M., "The Free Formulation for Nonlinear Finite Elements with Applications to Shells," Dr. Ing., Thesis, Div. of Structural Mechanics, Norwegian Inst. of Technology, Trondheim, Norway, 1986.

[32] Battini, J., and Pacoste, C., "On the Choice of the Linear Element for Corotational Triangular Shells," Computer Methods in Applied Mechanics and Engineering, Vol. 195, Nos. 44-47, 2006, pp. 63626377. doi:10.1016/j.cma.2006.01.007

[33] Rankin, C., and Brogan, F., "An Element Independent Co-rotational Procedure for the Treatment of Large Rotations," Journal of Pressure Vessel Technology, Vol. 108, No. 2, 1986, pp. 165-174. doi:10.1115/1.3264765

[34] Argyris, J., "An Excursion into Large Rotations," Computer Methods in Applied Mechanics and Engineering, Vol. 32, Nos. 1-3, 1982, 
pp. $85-155$.

doi: $10.1016 / 0045-7825(82) 90069-X$

[35] Geradin, M., and Cardona, A., Flexible Multibody Dynamics: A Finite Element Approach, Wiley, New York, March 2001.

[36] Shabana, A., Dynamics of Multibody Systems, Cambridge Univ. Press, New York, 2005.

[37] Zienkiewicz, O., and Taylor, R., The Finite Element Method, Butterworth-Heinemann, Maryland Heights, MO, 2005.

[38] Cook, R., Malkus, D., Plesha, M., and Witt, R., Concepts and Applications of Finite Element Analysis, Wiley, Hoboken, NJ, 2002.

[39] Subbaraj, K., and Dokainish, M., "Survey of Direct Time-Integration Methods in Computational Structural Dynamics: II. Implicit Methods," Computers and Structures, Vol. 32, No. 6, 1989, pp. 1387-1401. doi:10.1016/0045-7949(89)90315-5

[40] Jansen, K., Whiting, C., and Hulbert, G. M., "A Generalized- $\alpha$ Method for Integrating the Filtered Navier-Stokes Equations With a Stabilized
Finite Element Method," Computer Methods in Applied Mechanics and Engineering, Vol. 190, Nos. 3-4, 2000, pp. 305-319. doi:10.1016/S0045-7825(00)00203-6

[41] Hsiao, K., "Nonlinear Analysis of General Shell Structures by Flat Triangular Shell Elements," Computers and Structures, Vol. 25, No. 5, 1987, pp. 665-675. doi:10.1016/0045-7949(87)90159-3

[42] Wu, P., Ifju, P., Stanford, B., Sallstrom, E., Ukeiley, L., Love, R., and Lind, R., "A Multidisciplinary Experimental Study of Flapping Wing Aeroelasticity in Thrust Production," 50th AIAA/ASME/ASCE/AHS/ ASC Structures, Structural Dynamics, and Materials Conference, Palm Springs, CA, AIAA Paper 2009-2413, May 2009.
A. Palazotto Associate Editor 